\title{
Zika virus infects human testicular tissue and germ cells
}

\author{
Giulia Matusali, ${ }^{1}$ Laurent Houzet, ${ }^{1}$ Anne-Pascale Satie, ${ }^{1}$ Dominique Mahé, ${ }^{1}$ Florence Aubry, ${ }^{1}$ Thérèse Couderc, ${ }^{2,3}$ Julie Frouard, \\ Salomé Bourgeau, ${ }^{1}$ Karim Bensalah, ${ }^{4}$ Sylvain Lavoué, ${ }^{5}$ Guillaume Joguet, ${ }^{6}$ Louis Bujan, ${ }^{7}$ André Cabié, ${ }^{8}$ Cleide Avelar, ${ }^{9}$ \\ Marc Lecuit,, 2,3,10 Anna Le Tortorec, ${ }^{1}$ and Nathalie Dejucq-Rainsford ${ }^{1}$ \\ ${ }^{1}$ Université de Rennes, Inserm, École des hautes études en santé publique (EHESP), Institut de recherche en santé, environnement et travail (Irset) - UMR_S1085, Rennes, France. ${ }^{2}$ Institut Pasteur, Biology

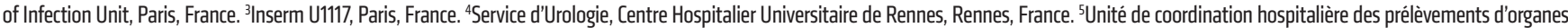 \\ et de tissus, Centre Hospitalier Universitaire de Rennes, Rennes, France. ${ }^{6}$ Centre Caribéen de Médecine de la Reproduction-CECOS CHU de Pointe-à-Pitre, Pointe-à-Pitre, France. ${ }^{7}$ Research Group on Human \\ Fertility EA 3694, University Paul Sabatier Toulouse III - CECOS, Hôpital Paule de Viguier, CHU Toulouse, Toulouse, France. ${ }^{8}$ Inserm Centre d'Investigation Clinique 1424, Centre Hospitalier Universitaire de \\ Martinique, and Service de maladies infectieuses, Centre Hospitalier Universitaire de Martinique, Fort de France, France. 'Department of Morphology, Federal University of Minas Gerais, Belo Horizonte, \\ Brazil. ${ }^{10}$ Paris-Descartes University, Department of Infectious Diseases and Tropical Medicine, Necker-Enfants Malades University Hospital, Paris, France.
}

Zika virus (ZIKV) is a teratogenic mosquito-borne flavivirus that can be sexually transmitted from man to woman. The finding of high viral loads and prolonged viral shedding in semen suggests that ZIKV replicates within the human male genital tract, but its target organs are unknown. Using ex vivo infection of organotypic cultures, we demonstrated here that ZIKV replicates in human testicular tissue and infects a broad range of cell types, including germ cells, which we also identified as infected in semen from ZIKV-infected donors. ZIKV had no major deleterious effect on the morphology and hormonal production of the human testis explants. Infection induced a broad antiviral response but no IFN upregulation and minimal proinflammatory response in testis explants, with no cytopathic effect. Finally, we studied ZIKV infection in mouse testis and compared it to human infection. This study provides key insights into how ZIKV may persist in semen and alter semen parameters, as well as a valuable tool for testing antiviral agents.

\section{Introduction}

Zika virus (ZIKV) is a teratogenic arthropod-borne flavivirus that recently emerged in the Pacific Islands (2007), Oceania (2013), and the Americas (2015). While ZIKV's primary mode of transmission is through mosquito bites, male-to-female sexual transmission has been reported by cohort studies $(1,2)$ and by case reports in non-endemic countries (3). Importantly, maleto-female sexual transmission in animal models was found to enhance viral dissemination in the female genital tract and transmission to the fetus (4-8). In humans, high viral loads and prolonged shedding of viral RNA (vRNA) and infectious virus (up to 1 year and 69 days, respectively) in the absence of viremia have been found in semen (9-11), strongly suggesting tropism of ZIKV for the male genital tract. Studies in immunodeficient mice evidenced high levels of ZIKV infection within the testis, leading to orchitis and impaired testosterone and sperm production (12-15). However, these mouse models do not reflect the pathophysiology in humans: unlike humans, mice only become infected following abrogation of type I IFN signaling and die of infection in most cases. This defective antiviral response may enhance the susceptibility and pathogenicity of ZIKV. In sharp contrast, ZIKV infection in macaque models either spared the testis or led to moderate infection, with no deleterious effects observed (16-18). Interestingly, a recent study on $15 \mathrm{ZIKV}$ infected men reported a lower total sperm count on day 30 after

Conflict of interest: The authors have declared that no conflict of interest exists Submitted: April 19, 2018; Accepted: July 24, 2018.

Reference information: J Clin Invest. 2018;128(10):4697-4710.

https://doi.org/10.1172/JCI121735. onset of symptoms compared with day 7, suggesting an effect of the infection on the testis or epididymis (19).

Here, by infecting with ZIKV testicular tissue explants from healthy donors, we show that ZIKV replicates and produces infectious viral particles in human testis. We found evidence of infection of a broad range of testicular cell types, including resident macrophages and the germ cell line, and confirmed the latter in patients' semen. Infection had no effect on basal testosterone and inhibin B production or overall cell viability ex vivo. ZIKV triggered a wide range of antiviral genes in human testes, but upregulation of types I, II, and III IFN was not observed, and proinflammatory response was minimal. Finally, our data on $\mathrm{IFNAR}^{-/-}$mice points to similarities and differences between mouse and human testis in response to ZIKV infection.

\section{Results}

$Z I K V$ replicates in human testicular tissue. Testis explants from 8 uninfected donors were exposed to ZIKV ex vivo and maintained in culture medium as previously described (20). We first assessed the replication rate of a ZIKV strain derived from the 2015 outbreak in the Americas by measuring viral release over 3-day culture periods on days 3, 6, and 9 after infection (p.i.). A significant increase in vRNA release rate was observed between days 3 and 6 (median $5.85 \times 10^{7}$ copies $/ \mathrm{ml}$ ) and 6 and 9 (median 8.28 $\times 10^{7}$ copies $/ \mathrm{ml}$ ) compared with days 0-3 p.i. (median $5.29 \times$ $10^{6}$ copies $/ \mathrm{ml}$ ) (Figure 1A), while vRNA was below the detection threshold in mock-infected testes (data not shown).

The ability of testes to produce infectious ZIKV particles was tested on reporter VeroE6 cells. A significant increase in supernatant infectivity was observed between days 0 and 3 (median $3 \times 10^{2}$ 

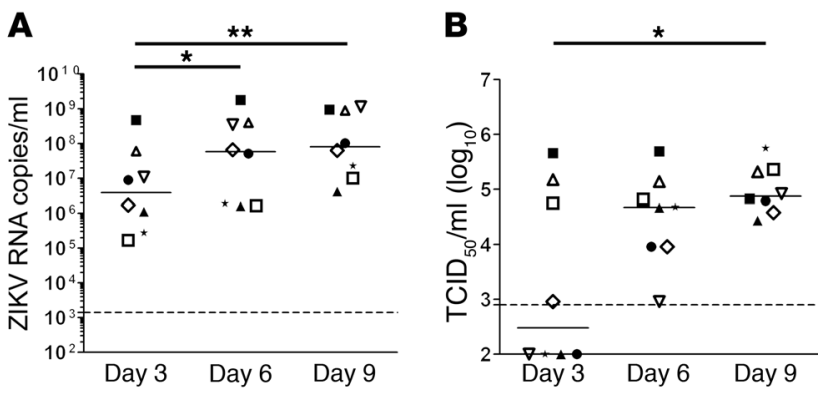

Figure 1. ZIKA virus replicates in human testicular tissue. Human testis explants from 8 donors were ex vivo infected overnight with $10^{5} \mathrm{TCID}_{50}$ (corresponding to $2.2 \times 10^{7}$ to $2.9 \times 10^{7}$ vRNA copies) from a low-passage ZIKV strain isolated in 2015 in the French Caribbean (MRS_OPY_Martinique_ PaRi-2015). Explants were thoroughly washed and cultured on inserts in 1 $\mathrm{ml}$ medium/well for 9 days, with media fully removed and changed every 3 days. Each of the time points (days 3, 6, 9) represents de novo viral release over a 3-day-culture period. (A) ZIKV RNA release over a 3-day culture period on days 3, 6, and 9 detected by RT-qPCR. (B) Viral titers determined by infectivity assay of 3-day culture period tissue supernatants on VeroE6 cells. Each symbol represents a different donor (same symbol/ donor throughout the figures). Dotted lines represent the detection limit of the assays. Mock-infected explants were always below detection level. Bars represent median. ${ }^{*} P<0.05,{ }^{*} P<0.01$ (Friedman-Dunn nonparametric comparison).

$\mathrm{TCID}_{50}[50 \%$ tissue culture infective dose $] / \mathrm{ml}$ ) and days 6 and 9 p.i. (median $7.50 \times 10^{4} \mathrm{TCID}_{50} / \mathrm{ml}$ ), demonstrating the infectivity of viral progeny (Figure 1B). The highest cumulative titer on day 9 (i.e., reflecting infectious viral production throughout culture) was $2 \times 10^{6}$ TCID $_{50} / \mathrm{ml}$ (Supplemental Figure 1; supplemental material available online with this article; https://doi.org/10.1172/JCI121735DS1), with a median of $3.16 \times 10^{5} \mathrm{TCID}_{50} / \mathrm{ml}$. Similarly, vRNA and infectious virion release rates increased during the culture of testis explants exposed to another ZIKV strain isolated during the 2013 outbreak in French Polynesia (Supplemental Figure 2).

Altogether, these data demonstrate that ZIKV efficiently infects and replicates in the human testis ex vivo, producing infectious viral particles.

ZIKV infects somatic and germ cells in human testis explants. To determine ZIKV's target cells in the human testis, we submitted mock- or ZIKV-infected testis explants to RNAscope in situ hybridization (ISH) using probes specific for ZIKV RNA (Figure 2, A-H; controls in Figure 2A and Supplemental Figure 3) and to IHC using an antibody against the nonstructural NS1 viral protein (Figure 2, $\mathrm{I}-\mathrm{M})$. Infected testes showed strong vRNA staining of the interstitial tissue cells and within the extracellular matrix bordering the seminiferous tubules, along with more diffuse staining in some interstitial areas (Figure 2, B-F). A weaker spotty staining was also observed inside a few seminiferous tubules (Figure 2, G and H), suggestive of association of the ZIKV with germ cells (Figure 2G) and Sertoli cells (Figure 2H). NS1 antibody (Figure 2, I-M) similarly labeled cells within the seminiferous tubule wall (Figure 2I) and the interstitium (Figure 2J), demonstrating ZIKV replication in these target cells. Within the tubules, different germ cell categories including spermatogonia (identified based on their position in the seminiferous epithelium, nucleus size, and distinctive morphological features) (Figure 2K) and a few Sertoli cells (identified based on distinctive nucleus shape) (Figure 2L) stained positive for NS1. Infected cells $\left(\mathrm{vRNA}^{+}\right.$or $\mathrm{NS}^{+}$) displayed similar localization at the different time points of infection (days 3, 6, and 9) for the $2 \mathrm{ZIKV}$ strains tested (Supplemental Figure 4 and data not shown).

To further identify the nature of the infected cells, we combined ISH for vRNA with fluorescence immunolabeling for specific cell markers and undertook quantification of infected cells in testicular tissue from 4 donors. Interstitial infected cells were primarily CD68/CD163 ${ }^{+}$testicular macrophages (median 12.7 cells $/ \mathrm{mm}^{2}$ ), and to a lesser extent CYP11A1 $1^{+}$Leydig cells (median 3 cells $/ \mathrm{mm}^{2}$ ) (Figure 3, A, B, and G). Staining for $\alpha$-smooth actin ( $\alpha$-SMA) demonstrated the infection of myoid peritubular cells bordering the seminiferous tubules (median 10 cells $/ \mathrm{mm}^{2}$ ) (Figure 3, C and G). Within the tubules, spotty fluorescent ZIKV staining close to the lumen histologically colocalized with late germ cells (Figure 3D). Such staining was also present at the base of the tubules, where colabeled DDX $4^{+}$early germ cells were evidenced (DDX4 being a specific marker expressed by most of the germ cells) (Figure 3E). Staining was not observed when a vRNA probe was used on mock-infected negative controls (Figure 3F). Infected cells in seminiferous tubules were mostly germ cells (median 11 cells $/ \mathrm{mm}^{2}$ ), while infected Sertoli cells represented a median of 3.5 cells $/ \mathrm{mm}^{2}$ (Figure $3 \mathrm{G}$ ).

Collectively these data indicate that ZIKV has tropism for germ cells and somatic cells within the human testis.

ZIKV replicates in human testicular germ cells in vitro and in vivo. We exposed freshly isolated seminiferous tubule cells to ZIKV to investigate their ability to produce infectious viral particles that might be released into semen. In 3 independent primary cultures, vRNA increased in cells from a median of $2.82 \times 10^{3}$ to $2.09 \times 10^{7}$ copies/ $\mu \mathrm{g}$ total RNA between 6 and 120 hours p.i. (Figure 4A). ZIKV RNA in culture supernatants significantly increased about $4 \log _{10}$ between 6 and 120 hours p.i. (median values of $1.26 \times 10^{4}$ and $5.01 \times 10^{7}$ copies/ml, respectively) (Figure $4 \mathrm{~B}$ ). Infectious virus titers also rose between 48 and 120 hours, reaching a median of $4 \times 10^{5}$ TCID $_{50} /$ $\mathrm{ml}$ and maximum titer of $4 \times 10^{6} \mathrm{TCID}_{50} / \mathrm{ml}$ (Figure $4 \mathrm{C}$ ). ZIKV replicated in $\mathrm{DDX}^{+}$germ cells, FSH receptor ${ }^{+}$Sertoli cells, and $\alpha-\mathrm{SMA}^{+}$peritubular cells (Figure 4D). ZIKV envelope (ZIKV-E) was detected in undifferentiated spermatogonia (MAGEA $-4^{+}$Stra8 ${ }^{-}$) and in MAGEA $-4^{+}$STRA $8^{+}$cells, corresponding to differentiated spermatogonia up to the preleptotene spermatocytes stage (Figure 4D).

To further explore the germ cells' productive infection and since primary testicular germ cells cannot be cultured without somatic support, we used the seminoma-derived germ cell line T-cam2, which displays characteristics of fetal germ cells (21). In 3 independent experiments, vRNA levels in T-cam 2 cells rose from below detection at 6 hours to a median of $6.31 \times 10^{5}$ copies/ $\mu$ g total RNA at 72 hours p.i., reaching a maximum of 1 $\times 10^{6}$ copies $/ \mathrm{ml}$ in culture supernatants (Supplemental Figure $5, \mathrm{~A}$ and B). ZIKV-E was evidenced in T-cam2 by immunofluorescence (Supplemental Figure $5 \mathrm{C}$ ). The production of infectious viral particles was evidenced in the 2 cultures showing the highest viral loads, with a maximum titer of $8.2 \times 10^{3} \mathrm{TCID}_{50} / \mathrm{ml}$ (Supplemental Figure 5D).

These findings were corroborated in vivo by analysis of the semen cell smear from 2 ZIKV-infected donors, in which we revealed the presence of $\mathrm{ZIKV}-\mathrm{E}^{+}$or $\mathrm{NS}^{+}$germ cells exfoliated from 

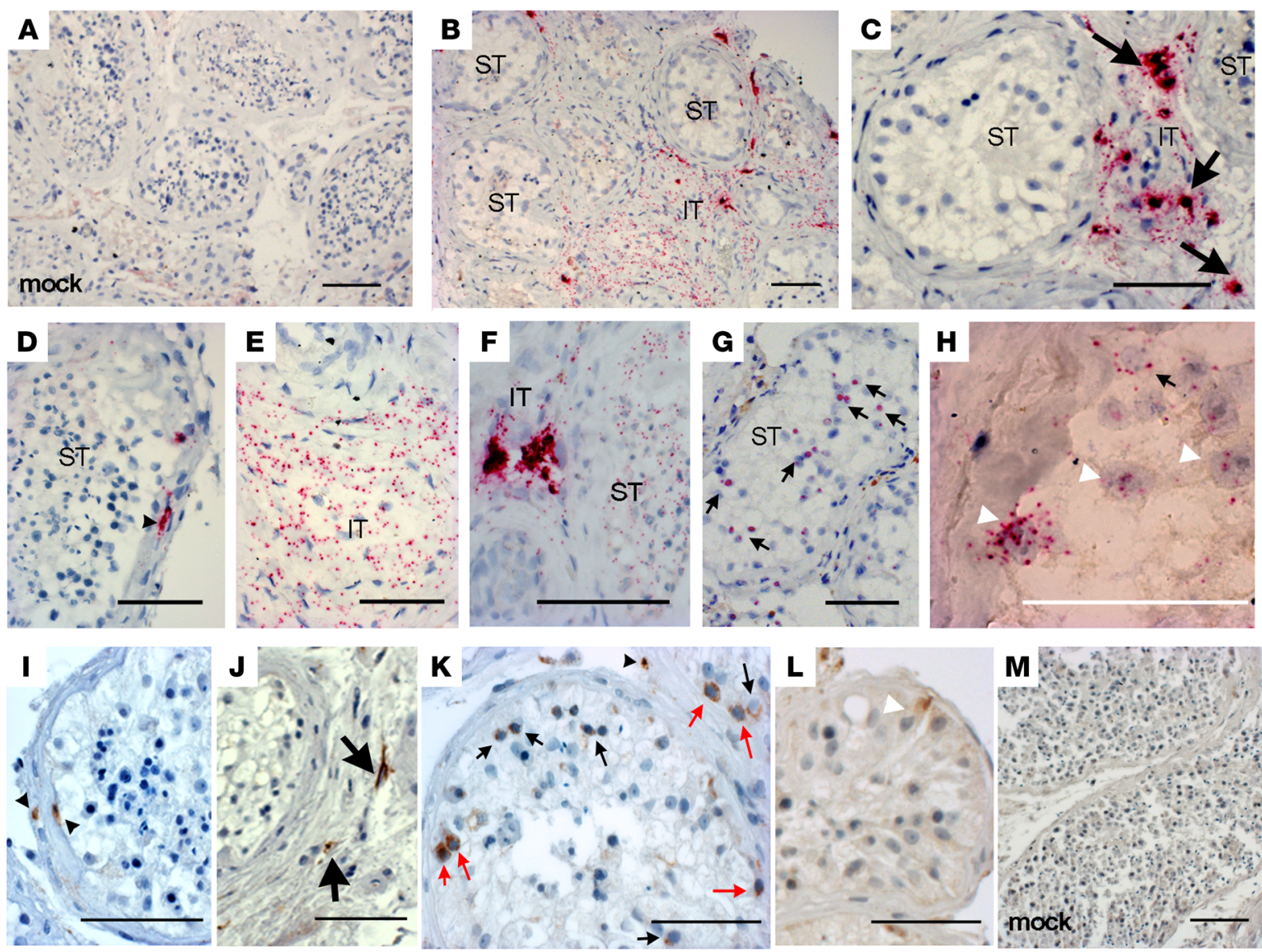

Figure 2. ZIKV infects somatic and germ cells in human testis explants. (A-H) Representative images of RNAscope ISH for ZIKV RNA in control mockinfected (A) and ZIKV-infected testis explants ( $n=8$ independent donors) after 6 days of culture (B-H). ZIKV RNA labeling was observed in the interstitial tissue (IT) of testis explants (B, C, E, and F), in cells bordering the seminiferous tubules (ST) (B and $\mathbf{D})$, and within seminiferous tubules (F-H). (I-M) Representative images of IHC staining of NS1-ZIKV performed on ZIKV-infected (I-L) and mock-infected (M) testis explants in culture for 6 days ( $n=8$ independent donors). Black arrowheads indicate infected cells in the extracellular matrix surrounding the seminiferous tubules. Thick arrows indicate infected cells in the interstitial tissue. Thin black arrows indicate infected germ cells. Thin red arrows indicate infected spermatogonia. White arrowheads indicate Sertoli cell nuclei. Black scale bars: $100 \mu \mathrm{m}$; white bar: $50 \mu \mathrm{m}$.

the testis 7 and 11 days after symptoms onset (Figure 4E). A subset of spermatozoa also labeled for ZIKV-E (Supplemental Figure 6).

Altogether, these data indicate that ZIKV replicates in human germ cells at different stages of differentiation and infects testicular germ cells in ZIKV-infected men.

ZIKV infection ex vivo has no major impact on human testis morphology or hormonal production. We next assessed the impact of ZIKV on human testis morphology, viability, and function during the ex vivo culture time frame. The tissue architecture and histology of infected testes were similar to those of mock-infected testes all throughout the culture period (Figure 5). In both infected and mock-infected testes, we observed conserved interstitial tissue (comprising groups of Leydig cells, mast cells, and blood vessels), seminiferous basement membrane of similar thickness (increased thickness being a sign of injury), and seminiferous tubules encompassing Sertoli cells and early and late germ cells (Figure 5, A and D). Caspase-dependent apoptosis evidenced by cleaved caspase-3 immunostaining was similar in infected and mock-infected testis and, as expected, primarily affected isolated germ cells (Figure $5 \mathrm{~B})$. Measurement of lactate dehydrogenase (LDH) release confirmed that the overall viability of the organ was not affected by the infection (Figure 5C). Testosterone concentrations were not different in infected versus mock-infected testes (Figure 5E), and expression of genes encoding steroidogenic enzymes was unmodified by ZIKV (Supplemental Figure 7A). Sertoli cells positively stained for the tight junction marker protein $\mathrm{ZO}-1$ in both infected and mock-infected testis until day 9 p.i., suggesting an intact barrier (Figure 5F). Inhibin B (a marker of Sertoli cell function) protein and mRNA levels showed no significant differences between infected and mock-infected testis up to day 9 p.i. (Figure 5G and Supplemental Figure 7B). Finally, levels of peritubular cells $\left(\mathrm{Acta}^{+}\right)$and early meiotic $\left(\mathrm{PGK}^{+}\right)$and late postmeiotic $\left(\mathrm{PRM}^{+}\right)$ germ cell transcripts were unchanged by the infection (Supplemental Figure 7B).

Overall, although actively replicating within the testis, ZIKV did not appear to affect testis morphology, induce cell death, or trigger any drastic effect on testis functions during the 9-day culture.

ZIKV triggers a broad antiviral response but no IFN upregulation and a minimal proinflammatory response in human testicular tissue. To investigate the immune response to ZIKV infection, we assessed the concentration of a panel of antiviral and proinflammatory cytokines (IFN- $\beta$, IFN- $\alpha 2$, IFN- $\lambda 1$, IFN- $\lambda 2 / 3$, IFN- $\gamma$, IL- $1 \beta$, 

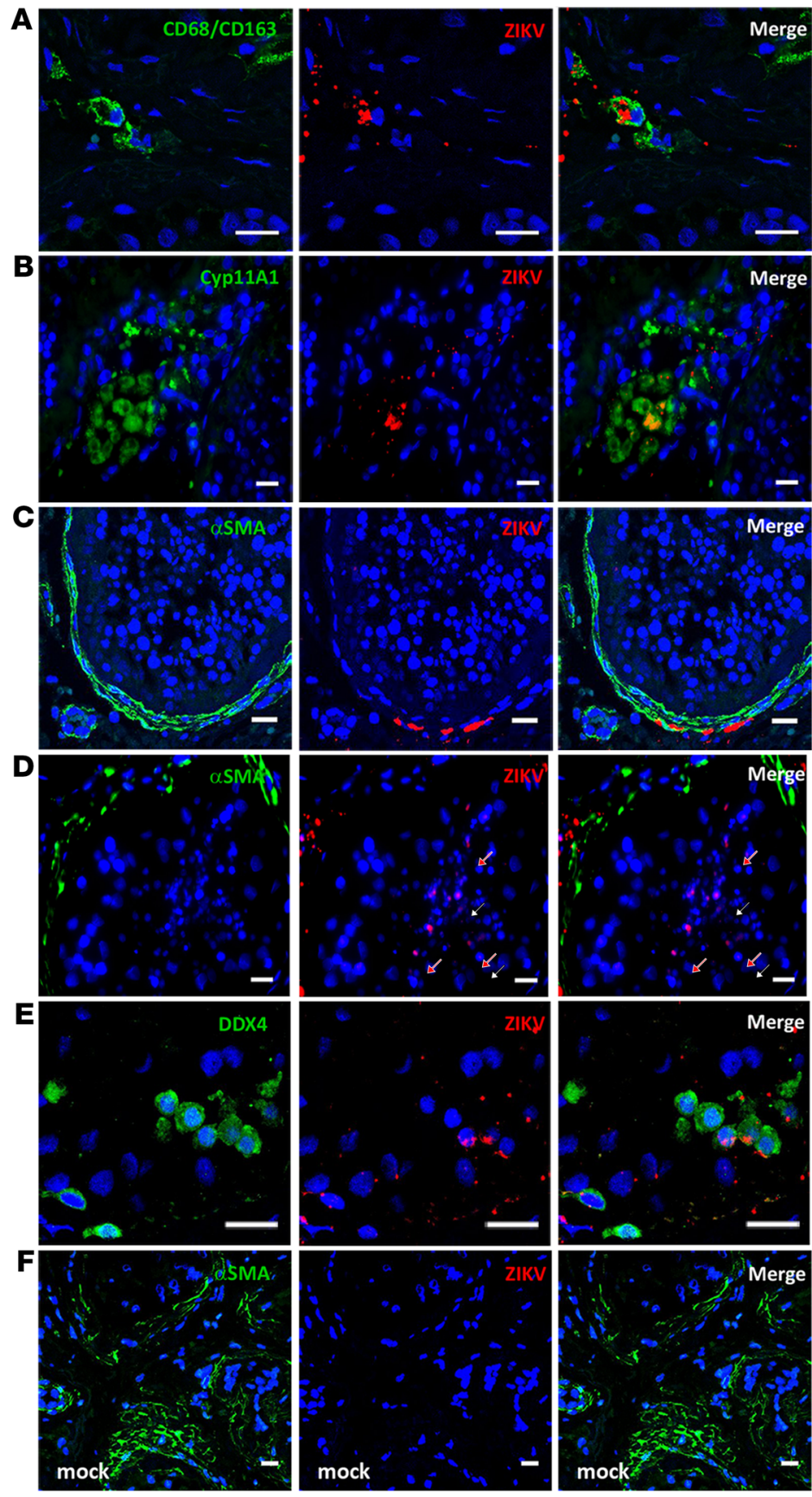

G
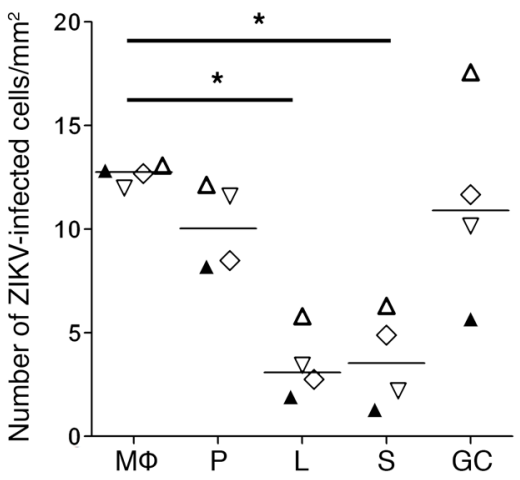

Figure 3. Characterization and quantification of ZIKVinfected human testicular cells ex vivo. RNAscope ISH for vRNA coupled with immunofluorescence for cell markers identified ZIKV RNA in CD68/CD163+ macrophages (A), Cyp11A1+ Leydig cells (B), $\alpha-S M A^{+}$peritubular cells (C), late germ cells localized near the lumen in seminiferous tubules (white arrows, round spermatids; red arrows, elongated spermatids) (D), and DDX4+ early germ cells (E). Staining for ZIKV was not observed in mockinfected testis (F). Nuclei are stained in blue. Scale bars: $20 \mu \mathrm{m}$. (C) Infected cells were quantified in at least 3 whole tissue sections from 4 testis donors (each represented by a different symbol) on day 9 p.i. M $\phi$, macrophages; P, peritubular cells; L, Leydig cells; S, Sertoli cells; GC: germ cells. Bars represent median. ${ }^{*} P<0.05$ (Friedman-Dunn nonparametric comparison).
IL-6, TNF- $\alpha$, IL-8, IL-12p70, CXCL10, IL-10, and GM-CSF) in testis explant supernatants. Type I, II, and III IFN concentrations in testis culture supernatants were unchanged by the infection on days 3 and 6 p.i. in 7 independent testis cultures tested (Figure 6A and Supplemental Figure 8). Among proinflammatory cytokines, only CXCL10 was significantly increased (Figure 6A and Supplemental Figure 8), and its induction positively correlated with vRNA load (Figure 6B).

We then analyzed the transcripts of 11 of these cytokines (IFN- $\beta$, IFN- $\alpha 1$, IFN- $\alpha 2$, IFN- $\alpha 4$, IFN- $\lambda 1$, IFN- $\lambda 2$, IFN- $\lambda 3$, IL-1 $\beta$, IL-6, TNF- $\alpha$, and CXCL10) by quantitative real-time PCR (RT-qPCR) on ZIKV-infected versus mock-infected testes. Type I, II, and III IFN transcripts in uninfected testis tissues were below the measure- threshold irrespective of infection (data not shown), while CXCL10 was increased (median fold change [FC] 43.4 on day 9, range 7.7-227.8) in the testis from 4 of 6 donors (Figure 6C).

Extending the analysis to a wider range of genes involved in pathogen sensing (RIG-I, MDA5), antiviral response (IFN- $\varepsilon$, IFI27, IFIT1, IFITM1, IRF7, ISG15, Mx1, Mx2, OAS1, OAS2, RSAD2), inflammation (CCR7, CD14, CD64, HLA-DR, MCSF), chemoattraction (CCL2, CCL5, CXCL1, CXCL2), and control of inflammation (IL-10, TGF- $\beta$, CD163, SOCS1, SOCS3), we observed the induction of a broad range of antiviral genes from day 3 onward in testis from 3 of 6 donors and on day 9 in 1 other donor (Figure $6 \mathrm{C}$ ).

A strong induction of ISG15 (FC 12.2, range 5.2-45.6), IFIT-1 (FC 12.8, range 9.7-29.6), OAS1 (FC 22.9, range 6.4-32.5), OAS2 (FC 
A
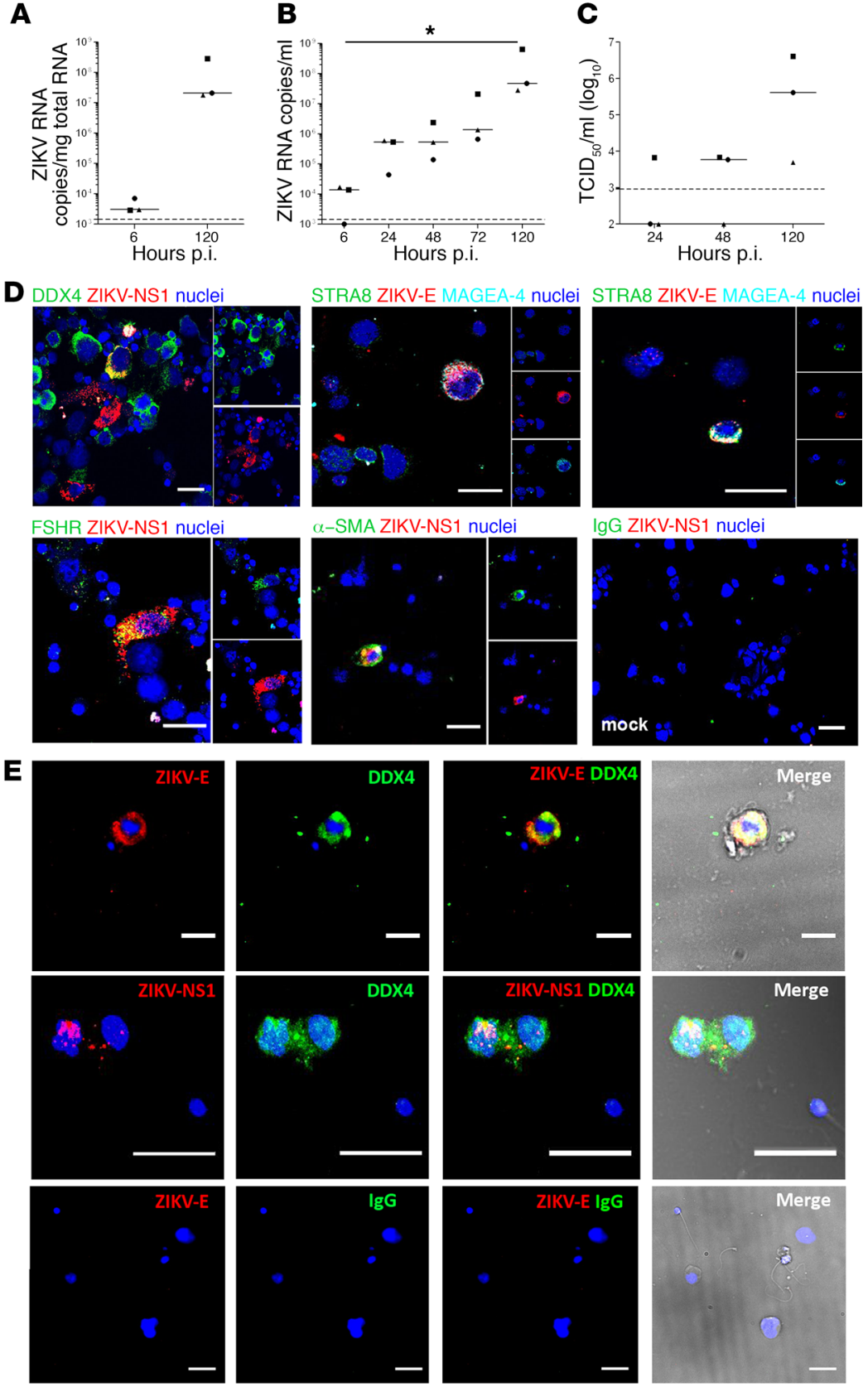

Figure 4. ZIKV replicates in human testicular germ cells in vitro and in vivo. (A-C) Primary testicular cells were infected with ZIKV (MOI of 1 , corresponding to $7.15 \times 10^{5} \mathrm{TCID}_{50} \mathrm{U} / \mathrm{ml}$ per 0.5 million cells). ZIKV RNA detected by RT-qPCR in cells (A) and culture supernatants (B). (C) Viral titers determined by infectivity assay of tissue supernatants on VeroE6 cells. Each dot represents an independent donor. Bars represent median values. Dotted lines indicate detection limit. ${ }^{*} P<0.05$ (Friedman-Dunn nonparametric comparison). (D) Immunofluorescence against ZIKV NS1 or ZIKV-E proteins combined with cell markers for all germ cells (DDX4) or specific germ cell types (STRA8, MAGEA-4), Sertoli cells (FSHR), and peritubular cells $(\alpha-S M A)$. Nuclei are stained in blue. (E) Detection of infected germ cells in semen from ZIKV-infected men. Immunofluorescence labeling of semen cell smears from 2 ZIKV-infected patients, one on day 7 (top row) and one on day 11 (middle row) after onset of symptoms. ZIKV-E or NS1 protein colabeled with the germ cell marker DDX4. Bottom panels show semen from a healthy individual stained with anti-ZIKV-E antibody and IgG isotype as a negative control. Nuclei are stained in blue. In the merge panels, brightfield images are included to visualize the cell's morphology. Scale bars: $20 \mu \mathrm{m}$.
7.1, range 4.2-21.6), $\mathrm{Mx} 1$ (FC 9.8, range 5.1-35.1), Mx2 (FC 9.5, range 3.7-25.3), and RSAD2 (FC 31.5, range 3.7-66.8) was measured on day 9 in these 4 donors, along with a more moderate induction of IFI27 (FC 3.1, range 2.2-10.6), IFITM1 (FC 1.8, range 1.5-6.2), IRF7 (FC 2.7, range 1.8-6), MDA5 (FC 1.9, range 1.6-8.5), and RIG1 (FC 2.8, range 1.8-9.4) (Figure 6C).

These genes correlated with one another in FC in expression on day 9, except for IFITM1 and MDA5 (Supplemental Figure 9). The fold increase on day 9 of a number of genes involved in antiviral response (RSAD2, IFIT1, ISG15, OAS1, OAS2, Mx1, Mx2, IFI27, IRF7), pathogen sensing (RIG1), and CXCL10 positively correlated with the level of infection (Figure $6 \mathrm{~B}$ ) in the corresponding testis supernatant on days 3 and 6 (Figure 6E and Supplemental Figure 9).

Finally, we assessed expression levels of all transcripts, including type I, II, and III IFN, at earlier time points $(4,18,48$ hours p.i.) in 2 testis explants and did not observe any upregulation (Supplemental Figure 10 and data not shown).

Altogether, these results are consistent with ZIKV infection inducing a broad antiviral and minimal proinflammatory response in the absence of detectable IFN stimulation in human testis explants.

Innate immune response to ZIKV infection in the testis from IFNAR ${ }^{-1-}$ mice. To support our hypothesis of a type I IFN-independent antiviral response induced by ZIKV in testis and compare our findings on ZIKV tropism and initial antiviral/proinflammatory responses in human testis explants with those in a widely used animal model, we analyzed the testis of type I IFN receptor-defective (IFNAR ${ }^{-/}$) mice using similar techniques and viral strain.

ZIKV RNA measurement in testes from IFNAR ${ }^{-/}$ mice infected for 5 and 9 days showed high viral loads in this organ (Figure 7A). Despite differences in intensity and sequence of infection, ZIKV tropism in the mouse testis in vivo was comparable overall to that in human testis ex vivo. On day 5 , testicular infection localized primarily within the interstitial tissue and peritubular cells, while seminiferous tubules were spared (Figure 7B). Colabeling of ZIKV RNA with cell markers showed infection of steroidogenic acute regulatory protein-positive $\left(\mathrm{STAR}^{+}\right)$Leydig cells and F4/80 + macrophages (Figure $7 \mathrm{C}$ ). On day 9 p.i. (a time at which death occurred in some mice), strong labeling for vRNA became prominent within Sertoli and germ cells (Figure 7B). We did not observe modifications of testicular morphology at these early time points, in agreement with previous studies $(12,22)$.

We next examined the induction of genes involved in antiviral response and inflammation in mouse testis (Figure 7D). Similar to human testis explants, and despite a lack of type I IFN signaling, a strong induction of ISG15 (FC 11.7, range 7.7-15.6 on day 5 and FC 7.4, range 4.7-15.1 on day 9), RSAD2 

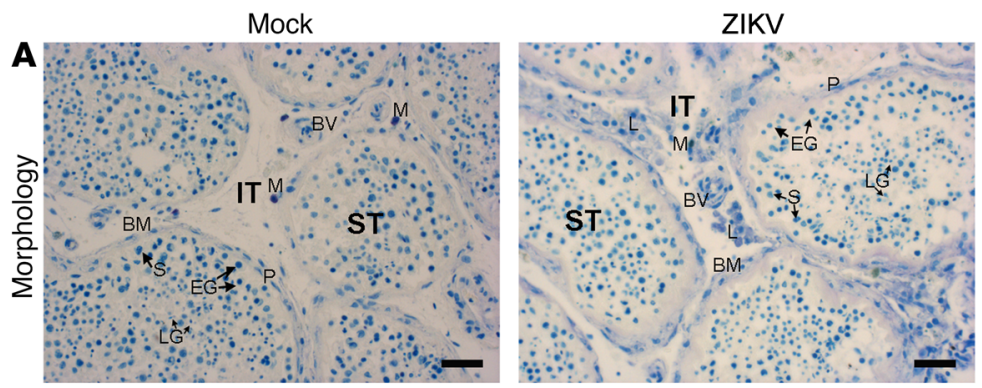

IT: interstitial tissue

ST: seminiferous tubules

BV: blood vessel

BM: basal membrane

L: Leydig cells

S: Sertoli cells

$\mathrm{P}$ : peritubular cells

M: mast cells

EG: early germ cells

LG: late germ cells
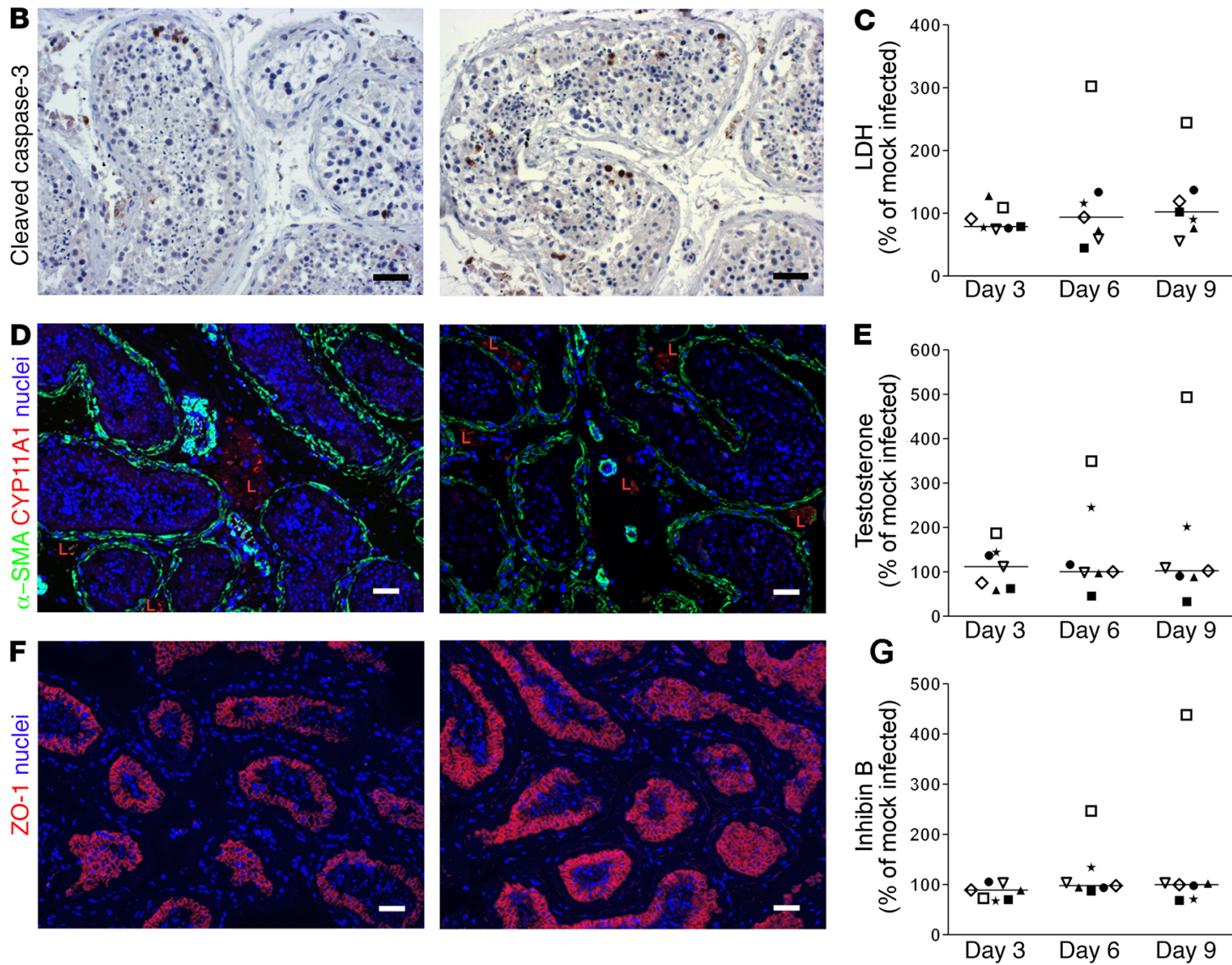

Figure 5. ZIKV infection ex vivo does not alter human testis explant morphology, cell viability, or hormonal production. (A) Toluidine histological staining of testis explants, shown here for mock- infected (left) and ZIKV-infected (right) testis explants on day 6 p.i. (B) Cleaved caspase-3 IHC to detect apoptotic cells in mock (left) and ZIKV-infected (right) testis explants, shown here for day 6 p.i. (C) LDH release in testis supernatant expressed as percent of mock-infected explants on the corresponding day of culture. (D) Immunofluorescence colabeling of peritubular ( $\alpha$-SMA) and Leydig (CYP11A1) cells, shown on tissue sections on day 6 p.i. for mock- (left) and ZIKV-infected (right) explants. Nuclei are stained in blue. (E and G) Testosterone and inhibin B release in testis supernatants expressed as percent of mock-infected explants on the corresponding day of culture. (F) Immunofluorescence labeling of Sertoli cell tight junction-associated protein ZO-1 in tissues sections for mock- (left) and ZIKV-infected (right) explants, shown on day 6 p.i. Nuclei are stained in blue. Scale bars: $50 \mu \mathrm{m}$. C, E, and G: each symbol represents a different donor; horizontal bars represent median values.

(FC 24.6, range 13.7-45.6 on day 5 and FC 10.1, range 2.21-30.39 on day 9), IFIT1 (FC 36.0, range 19.5-42.6 on day 5 and FC 10.1, range 6.2-27.3 on day 9), and CXCL10 (FC 17.5, range 9.4-39.2 on day 5 and FC 9.0, range 4.2-14.6 on day 9) was detected in infected mice testis (Figure 7D). In contrast to human testis, Mx1, MDA5, and RIG1 were not induced on either day 5 or day 9 (Figure 7D). These results suggest that ZIKV induces a type I IFN signalingindependent antiviral response in both humans and mice. In contrast to human testis, in which CXCL10 was the only proinflammatory gene increased by the infection, TNF- $\alpha$ (median FC 48.0, range 32.6-122.6 on day 5 and median FC 6.9, range 2.6-12.3 on day 9), IL-1 $\beta$ (median FC 7.7, range 1.8-13.8 on day 5 only), and IL-6 (median FC 10.7, range 4.9-27.5 on day 5 only) were upregulated in infected mouse testis (Figure 7D), while IFN- $\gamma$ (produced by NK and $\mathrm{T}$ cells) was maximally increased on day 9 (median FC 11.2, range 6.2-30.8) over day 5 (median FC 6.2, range 4.0-12.7). IFN- $\beta$ was the most dramatically stimulated innate immune gene on day 5 (median 236.7, range 130.2- to 353.4-fold), while IFN- $\alpha 1,-2$, and -4 genes were modestly and transiently upregulated on day 5 , and IFN- $\lambda 2 / 3$ mRNA levels were unchanged (Figure 7D). When look- 
A

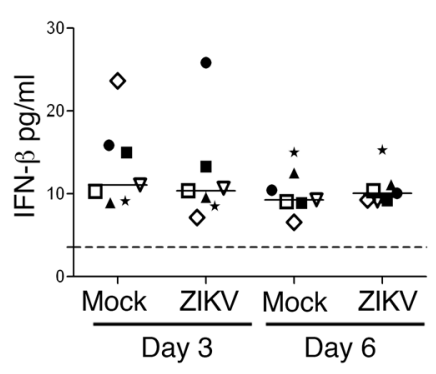

B

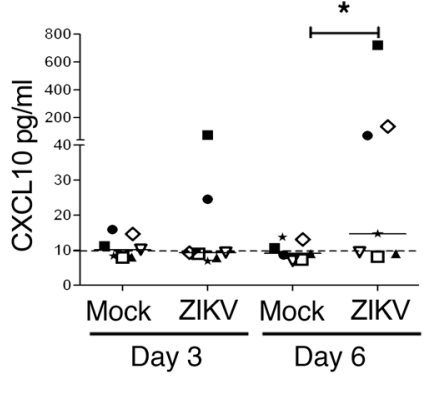

$>3$

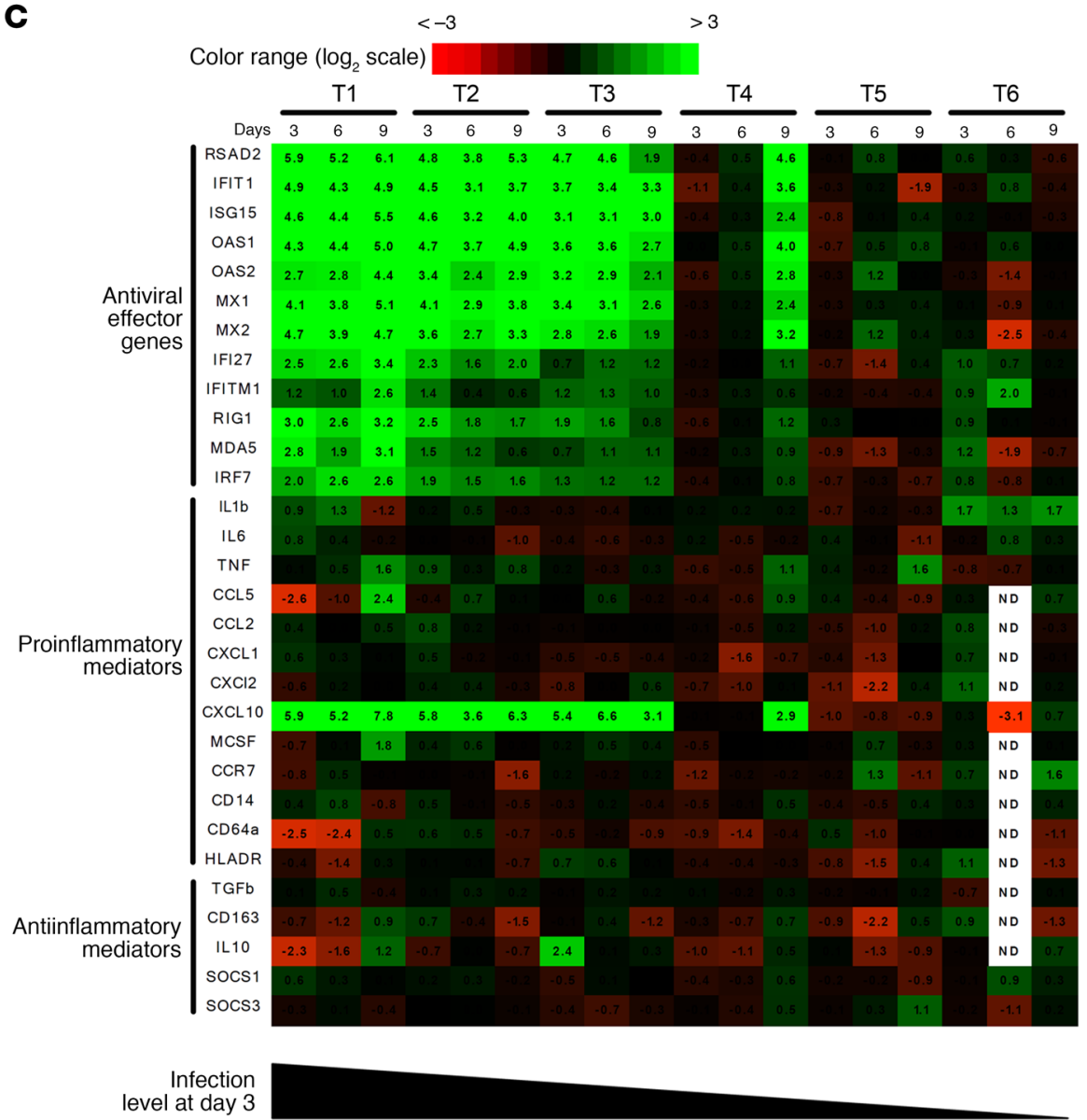

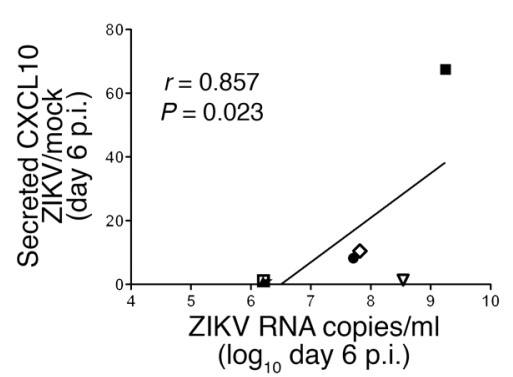

D

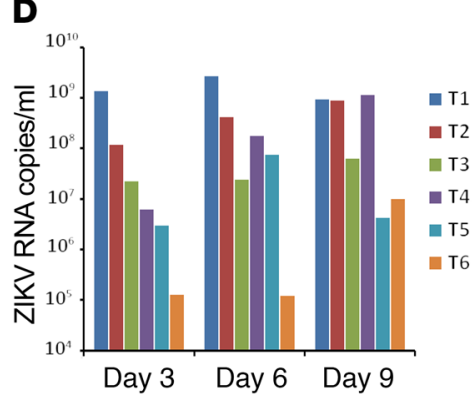

$\mathbf{E}$

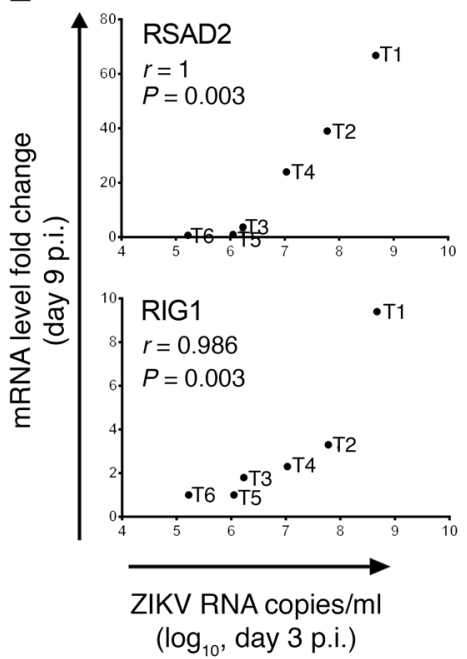

Figure 6. ZIKV triggers a broad antiviral response but no IFN upregulation and a minimal proinflammatory response in human testicular tissue. (A) Levels of IFN- $\beta$ and CXCL10 measured by flow cytometry-based multiplex assay in mock-infected and ZIKV-infected human testis explant supernatants. Each symbol represents a different donor. Bars represent median values. ${ }^{*} P<0.05$ (Friedman-Dunn nonparametric comparison). (B) Correlation between secreted CXCL10 induction in ZIKV-infected versus mock-infected explants and ZIKV RNA level in culture supernatant on day 6 p.i. (Spearman's nonparametric test). (C) Innate immune gene expression determined by RT-qPCR in testis explants from 6 donors (T1-T6) infected with ZIKV for 3, 6, and 9 days $(d 3, d 6, d 9)$. Heatmap shows $\log _{2}$-transformed expression ratios between ZIKV-infected and time-matched mock-infected controls. Green indicates upregulation and red downregulation of mRNA compared with controls. Type I and II IFN mRNAs were below the quantification threshold (data not shown). (D) Viral loads in supernatants of the testis explants analyzed in C. (E) Examples of correlation between gene fold expression on day 9 and the level of infection on day 3 p.i. (Spearman's nonparametric test). Other correlations are shown in Supplemental Figure 9.

ing at markers of immune cell subtypes, we observed a transient increase in transcripts encoding the myeloid cell marker CD14 on day 5, whereas transcripts encoding CD3 (T cell marker) and CD8 (cytotoxic $\mathrm{T}$ cell marker) were maximally upregulated on day 9 , in line with the IFN- $\gamma$ expression pattern. The markers for B cells (CD19 and CD20), regulatory T cells (FoxP3), and macrophages (CD68) were unchanged, while CD4 (expressed by Thelper and myeloid cells) was downregulated (Figure 7D). The infiltration of $\mathrm{T}$ lymphocytes in infected mouse testis was confirmed by CD3 immunostaining and quantification of positive cells (Supplemental Figure 11), further demonstrating mouse testis inflammation. Overall, the induction of an antiviral response in human and $\mathrm{IFNAR}^{--}$mice testis supports the existence of a type I IFN signaling-independent response to ZIKV infection in testis. 
A

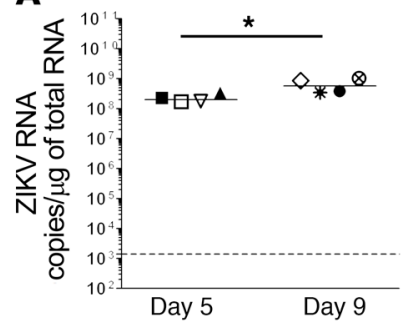

B

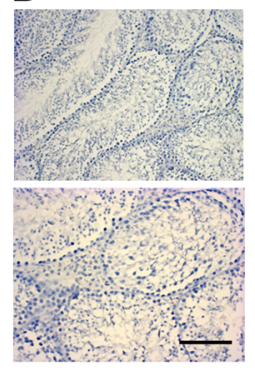

Mock

\section{C}
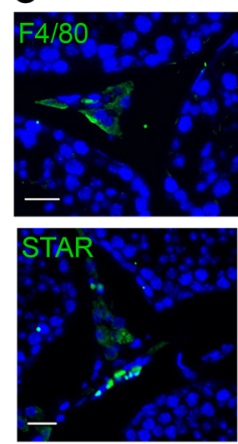

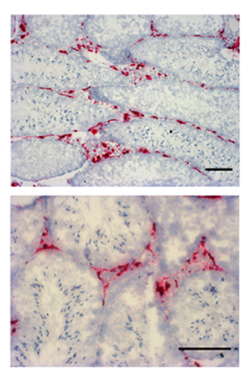

Day 5
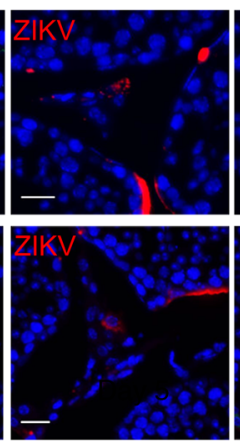

D

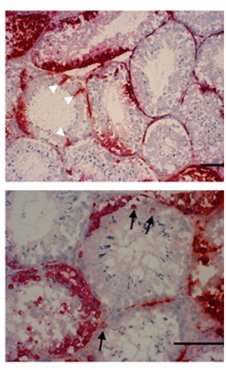

Day 9
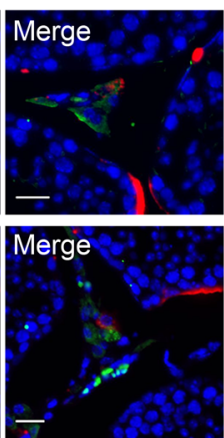

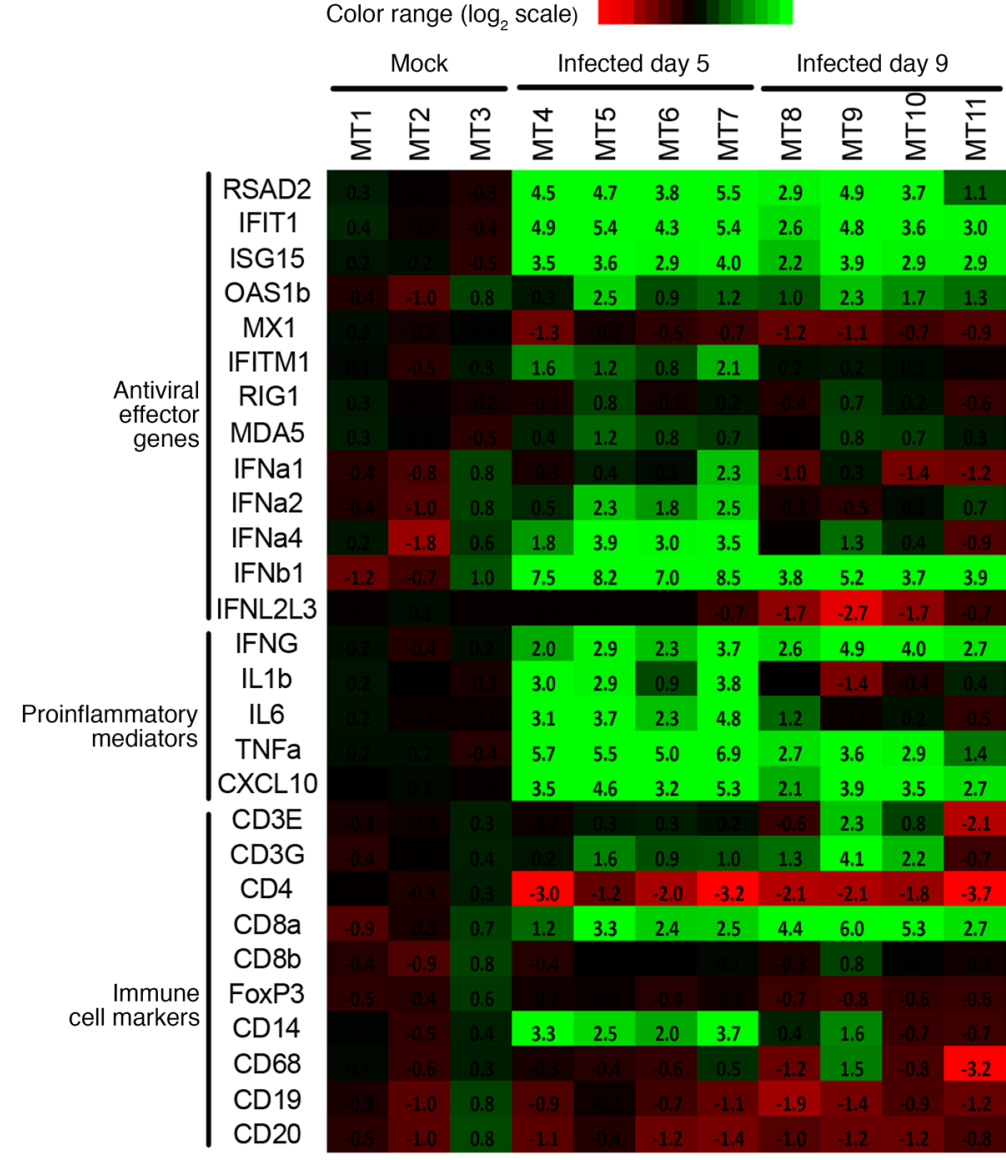

Figure 7. Innate immune response to ZIKV infection in testis from IFNAR ${ }^{-/-}$mouse. (A) VRNA measured by RT-qPCR in testis from mice infected with ZIKV for 5 or 9 days ( $n=4$ animals/group). Each dot represents one animal, and horizontal bars represent the median. The dotted line indicates the limit of detection. Levels in testis from mock-infected mice $(n=3)$ were below the detection threshold (data not shown). ${ }^{*} P<0.05$ (Mann-Whitney $U$ test, nonparametric comparison). (B) Detection of ZIKV RNA by RNAscope ISH in testis tissue sections from mice mock-infected or on day 5 or day 9 after infection. White arrowheads indicate Sertoli cells; thin black arrows indicate germ cells. Scale bars: $100 \mu \mathrm{m}$. (C) RNAscope ISH for ZIKV RNA coupled with immunofluorescence for cell markers identified ZIKV RNA in F4/80+ macrophages and STAR+ Leydig cells. Nuclei are stained in blue. Scale bars: $20 \mu \mathrm{m}$. (D) Expression of a range of innate immune genes and of genes encoding immune cell markers was determined by RT-qPCR in testis from 3 mock-infected mice (mouse testis MT1-MT3)

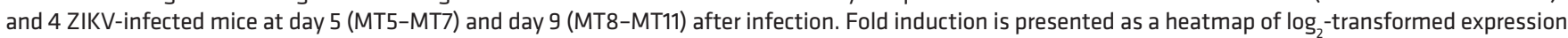
ratios to the average expression level in mock-infected mice. On the scale bar, green indicates upregulation and red, downregulation.

\section{Discussion}

We show that Asian ZIKV replicates in the human testis ex vivo. Infected somatic cells within testis explants were mostly macrophages and peritubular cells, while smaller numbers of Leydig and Sertoli cells were observed. Considering the relative proportion of these different cell types in human testicular tissue (approximately 1 macrophage for 10 Leydig cells, 36 peritubular cells, 40 Sertoli cells, and 400 germ cells) $(23,24)$, macrophages are likely the cell type most susceptible to ZIKV infection within the testis. Importantly, we demonstrate that ZIKV replicates within testicular germ cells, from stem cell (spermatogonia) to spermatozoa precursors (spermatids). In agreement with this finding, Robinson et al. recently reported on the infection of male germ cells after 3-day exposure of human seminiferous tubules to African ZIKV (25). Our detection of infected germ cells in semen from ZIVK- infected men confirms these in vitro and ex vivo findings. The presence of ZIKV in ejaculated spermatozoa adds to previous findings of ZIKV antigen, RNA, and infectious particles in spermatozoa $(19,26)$. Spermatozoa and immature germ cells may be infected during epididymal transit (duration 1-21 days) or within the testis. To infect these cells, the virus has to cross the bloodtestis barrier formed by Sertoli cell tight junctions. Direct infection of Sertoli cells is supported by our results in primary cells and those of other researchers in commercial Sertoli cells $(27,28)$. Sertoli cells were shown to release ZIKV particles on their adluminal side, whereas tight and adherens junction protein expression was not altered by infection (27). In agreement with these data, the ZO-1 labeling we observed in human explants suggested intact Sertoli cell barrier despite infection. In contrast, Sertoli cell exposure to inflammatory mediators produced by ZIKV-infected 
blood-derived macrophages (the phenotype of which differs from that of antiinflammatory testicular macrophages) altered their barrier function (27). Thus, the alteration of the blood-testis barrier by testis-infiltrating macrophages may provide an additional way for the virus to reach seminal lumen and late germ cells. Altogether, our data show that ZIKV replicates in germ cells and suggest that the virus might be able to bypass the blood-testis barrier by infecting Sertoli cells.

In our ex vivo model, release of testosterone and inhibin B was not modified by infection, nor were their related gene expression. This is not a limitation of our culture system, since it is successfully used to assess the hormonal production of human testis (29). This lack of effect might be linked to low infection levels of Leydig and Sertoli cells ex vivo, as supported by a relatively low number of infected cells. In ZIKV-infected mouse testis, testosterone and inhibin $\mathrm{B}$ levels were preserved on day 7 p.i., while testis integrity was maintained, whereas they decreased after orchitis $(12,13)$, suggesting that inflammation rather than testis infection caused altered hormone secretion. In a 4-month follow-up of a cohort of 15 ZIKV-infected men, testosterone levels were not significantly affected, whereas slightly lower inhibin B levels were reported on day 7 after onset of symptoms compared with later time points (19). Such transient imbalance of reproductive hormones can be related to fever or other systemic effects (30). However, we did not study the effect of ZIKV on LH- and FSH-stimulated hormone secretion, and a systemic impact on testicular hormones in vivo cannot be ruled out. Moreover, we cannot exclude effects at the single-cell level that cannot be detected when analyzing the whole tissue.

Our findings suggest that ZIKV could affect sperm production. Besides germ cell infection, somatic cell infection might disrupt the paracrine control of spermatogenesis $(31,32)$, and the infection of the contractile peritubular cell (33) might decrease the expulsion of spermatozoa from tubules into the epididymis. Infected men showed a decrease in sperm count and an increase in spermatozoa abnormalities during the 2 months after clinical onset $(19,34)$. Our results suggest that direct infection of germ and/or testicular somatic cells might be involved in such altered sperm parameters, although fever and/or immune response could be involved $(35,36)$.

The infection had no significant effects on testis explant morphology or viability. This is in contrast to findings in mouse models, where damaging effects of ZIKV infection on testis became evident after leukocyte infiltration (12-14). Since the ex vivo testis model lacks the presence of an intact immune system, we cannot rule out an effect of acquired immunity on the testis from ZIKVinfected men. However, testicular atrophy or orchitis has not been reported in clinical cases or nonhuman primate studies, and immune infiltrations were not observed in the latter (16-18), suggesting the absence of massive inflammation. The lack of cell death induction in our ex vivo model could be linked to culture conditions (e.g., viral strains/doses used, duration of infection) or to the limited number of infected cells. However, it might also reflect the ability of the virus to replicate in testicular cells in a noncytopathic manner. No or minimal cytopathic infection and persistence of ZIKV has been reported in different cell types, including human placental macrophages (37), brain microvascu- lar endothelial cells (38), and, recently, male mouse germ cells (25). Absence of cytopathic effect was also reported in Sertoli cells infected with ZIKV $(27,39)$. In contrast, high cytotoxicity was reported in ZIKV-infected human testis organoids (40). However, in this organoid system, the architecture of the testis is not preserved and physiological cellular interactions is lost. We hypothesize that nonlytic infection, in combination with evasion from immune responses, may allow viral persistence in the human testis.

A broad range of antiviral genes was induced by ZIKV in testis explants. Several of these classically defined IFN-stimulated genes (ISGs), such as ISG15 (41), RSAD2 (42), IFITM1 (43), OAS1 (44), Mx1 (45), and IFIT gene family members (46), have an inhibiting activity on flaviviruses and/or ZIKV replication. Most interestingly, ZIKV did not increase type I, II, or III IFN secretion by testicular explants, and their transcripts consistently remained below detection at all time points. A level of IFN production below the sensitivity of our assays, or active inhibition of IFN production by ZIKV, could explain these results. Thus, ZIKV nonstructural proteins inhibit different steps of the type I IFN induction cascade $(47,48)$. Alternatively, the absence of IFN upregulation in testis explants might reflect a specificity of this immunosuppressed organ, in which sustained high concentrations of type I IFN trigger germ cell apoptosis and sterility (49). Detection of ISG overexpression in testis from ZIKV-infected $\mathrm{IFNAR}^{-/}$mice further suggests IFN-independent induction of ISGs in the testis. The increased level of RIG1 mRNA in infected testis suggests it may be involved in the direct induction of ISGs, although other effectors could be in play (50). However, the broad induction of ISGs may fail to control ZIKV replication in the testis in the absence of increased type I IFN secretion to amplify and stabilize the antiviral response. In contrast to its effects on antiviral genes, ZIKV infection of the human testis did not affect any of the classic proinflammatory cytokines, except for CXCL10, secretion of which was modestly increased. Interestingly, the level of antiviral transcripts on day 9 p.i. positively correlated with the level of infection on days 3 and 6, suggesting that the initial level of infection influenced the intensity of the antiviral response. Accordingly, antiviral genes were not induced in explants showing lower levels of infection. Thus, host factors other than those we have studied may play a role in susceptibility to ZIKV. Altogether, our results indicate that ZIKV induces a broad induction of antiviral effectors but no IFN upregulation and minimal proinflammatory response in ex vivo infected human testis. We hypothesize that such innate immune response, along with a lack of cytopathic effect, might facilitate the persistence of ZIKV for extended periods in the testis and contribute to the prolonged release of ZIKV in semen.

Animal models are crucial for mechanistic studies and the in vivo testing of antiviral strategies. Cross-validation with human data is essential to assess their similarities and differences. Discrepant results on the interstitial $(13,22,51)$ and/or intratubular localization of ZIKV $(12,14,52,53)$ were reported in mouse testis from IFN signaling-deficient mice. Our results in the $\mathrm{IFNAR}^{-/-}$ mouse model reconciled these results, since ZIKV infection was exclusively located in interstitial and peritubular cells on day 5 p.i., whereas by day 9 the infection had progressed to the seminiferous tubules, where it became prominent. In human testis, seminiferous tubule cell infection was consistently weaker than that in the 
mouse and that in human interstitial cells. This was not modified when the infective viral dose was increased 10 times (data not shown). The difference in seminiferous tubule infection levels in mice versus humans might reflect differences in the susceptibility of mouse versus human Sertoli and germ cells to ZIKV infection and/or in their innate immune response.

We found evidence of induction of several ISG mRNAs in testis from $\mathrm{IFNAR}^{-/-}$mice, indicating type I IFN signalingindependent induction, which corroborates our results in human testis explants. Interestingly, in contrast to the human testis, the pathogen sensor RIG1 was not upregulated in the mouse testis, which may suggest a different sensing mechanism. Also unlike in human testis, type I IFNs and a number of proinflammatory genes were upregulated in mice. We previously showed that unlike their rodent counterparts, which produced large amounts of IFN, primary human Leydig cells did not produce IFN in response to paramyxovirus infection or double-stranded RNA stimulation $(54,55)$. This key difference between mouse and human testis in terms of IFN production may explain why in IFN signaling-competent mice, ZIKV tropism has been reported as being essentially restricted to germ cells (25), whereas a broad tropism for both somatic and germ cells is observed in human testis explants and in $\mathrm{IFNAR}^{-/-}$mice. Indeed, we previously showed in rodents that meiotic and postmeiotic male germ cells lack the functional type I IFN receptor (49) and do not express ISGs after viral or IFN stimulation, unlike testicular somatic cells $(56,57)$. Differences in antiviral (e.g., sensing pathways and ISG induction patterns) and proinflammatory immune responses in human versus mouse testis may explain the testis pathogenicity observed in type I IFN signaling-deficient mouse models (along with differences in infection levels). Differences in type I IFN upregulation following ZIKV infection may also explain the restricted tropism of ZIKV in testis from immunocompetent mice (25) when compared with human testis explants. Whether these differences derive from intrinsic differences between human and mouse testicular cells, differential escape mechanisms mediated by ZIKV (e.g., specific counteracting of type I IFN by ZIKV in human cells but not in immunocompetent mouse cells), or ex vivo/in vivo differences (e.g., infiltrating cell proinflammatory activity) requires further investigation.

To date, ZIKV is the only arbovirus known to be sexually transmitted within the human population. RNA from other arboviruses such as dengue, yellow fever, and chikungunya viruses were recently evidenced in semen from infected men for a prolonged period (5860), but no cases of sexual transmission have been documented so far. Dengue virus did not productively infect/alter testicular cells in mouse models $(12,13,25,51)$ and poorly infected human Sertoli cells. Although the neurotropic West Nile virus (WNV) replicated to levels similar to ZIKV in a Sertoli cell line (27), testis from men with neuroinvasive WNV tested negative, except for one immunosuppressed patient (61). Interestingly, Japanese encephalitis virus, another mosquito-borne neurotropic flavivirus, infects boar testis and semen for a long period of time, disrupts spermatogenesis, and can be transmitted through semen (62).

Of note, other male genital organs may be involved in ZIKV shedding in human semen, as suggested by prolonged sexual transmission of ZIKV from vasectomized men (63), and by ZIKV replication in human prostate cell lines and cell line-based organoids (64). Interestingly, we recently demonstrated in SIV-infected cynomolgus macaques that depending on the individuals, different male genital organs may be the source of the virus in semen (65). Nevertheless, the significant reduction in ZIKV titers and shorter infectivity window in semen from vasectomized mice indicate the importance of testis/epididymis contributions to infectious virus shedding (4).

In conclusion, we demonstrated that ZIKV replicates in the human testis ex vivo and infects a range of somatic cells and germ cells. Replication of ZIKV in testicular germ cells was evidenced in semen from ZIKV-infected men, along with ZIKV association with spermatozoa. ZIKV had no major deleterious effect on the morphology or hormonal production of the human testis in culture. Despite a broad induction of antiviral genes, absence of IFN upregulation and minimal proinflammatory response of the human testis ex vivo along with the lack of ZIKV cytopathic effect on testicular cells might favor the prolonged ZIKV infection observed in this organ and account for the absence of orchitis in men infected by ZIKV. Overall, our results suggest that ZIKV infection of the human testis may be involved in the persistence of the virus in semen and in altered semen parameters. These results call for further investigation of the impact of ZIKV on the reproductive health of ZIKV-infected men and warn against the potential horizontal and vertical transmission of ZIKV through the infected germ line. Finally, the ex vivo model of ZIKV infection of the human testis we developed provides a valuable tool for the testing of antiviral agents.

\section{Methods}

Cells lines and viruses. Asian Zika virus strains isolated during the 2015 outbreak in the French Caribbean (MRS_OPY_Martinique_PaRi-2015, passaged once in Vero cells) and during the 2013 outbreak in Polynesia (H/PF/2013, passaged 3 times in Vero cells) were obtained from the European Virus Archive (EVA) and further propagated in VeroE6 cells for 2 additional passages. VeroE6 cells (African green monkey kidney epithelial cells) were maintained in DMEM supplemented with $10 \%$ FCS, glutamine $(2 \mathrm{mM})$, and $1 \%$ penicillin/streptomycin at $37^{\circ} \mathrm{C}$ with $5 \% \mathrm{CO}_{2}$ (all reagents from Gibco). To produce viral stocks, VeroE6 cells were infected at an MOI of 0.01 in serum-free medium for 2 hours, and then complete medium was added to reach a final serum concentration of $5 \%$. When cytopathic effect was evident, supernatant was centrifuged, filtered $(0.45 \mu \mathrm{m})$, aliquoted, and frozen at $-80^{\circ} \mathrm{C}$. The human testicular germ cell tumor (seminoma) cell line Tcam-2 (66) was provided by Janet Shipley (Institute of Cancer Research, London, United Kingdom).

Organotypic culture of human testis explants and infection. Testes were dissected into 3- $\mathrm{mm}^{3}$ sections transferred onto 24-well plates (2 sections/well) containing $500 \mu \mathrm{l}$ medium (DMEM/F12 supplemented with $1 \times$ nonessential amino acids, $1 \times$ ITS (human insulin, human transferrin, and sodium selenite), $100 \mathrm{U} / \mathrm{ml}$ penicillin, $100 \mu \mathrm{g} /$ $\mathrm{ml}$ streptomycin, $10 \% \mathrm{FCS}$, all from Gibco) in the presence or absence of $10^{5} \mathrm{TCID}_{50} \mathrm{ZIKV}$ (corresponding to $2.2 \times 10^{7}$ to $2.9 \times 10^{7}$ vRNA copies for MRS_OPY_Martinique_PaRi-2015 and $8 \times 10^{7}$ vRNA copies for $\mathrm{H} / \mathrm{PF} / 2013)$. After overnight incubation, tissue fragments were washed 3 times with PBS and transferred onto a polyethylene tere- 
phthalate insert ( $3 \mu \mathrm{m}$ high-density pores) in 12-well plates containing $1 \mathrm{ml}$ medium. Eight hours later, the medium was changed again to further wash away potential residual virus input (time 0 for sample collection). For each experimental condition, a minimum of 2 wells were tested. The culture was maintained up to 9 days p.i. in a humidified atmosphere containing $5 \% \mathrm{CO}_{2}$ at $37^{\circ} \mathrm{C}$, with medium collected and fully changed every 3 days, in order to thoroughly wash input virus and assess viral production dynamic. Media were stored frozen at $-80^{\circ} \mathrm{C}$ for vRNA and titer measurement. Tissue fragments were either fixed in neutral buffered $4 \%$ formaldehyde or frozen and stored at $-80^{\circ} \mathrm{C}$.

Isolation and infection of testicular cells. Testis fragments were incubated in digesting medium $(2 \mathrm{mg} / \mathrm{ml}$ hyaluronidase, $2 \mathrm{mg} / \mathrm{ml}$ collagenase I, $20 \mu \mathrm{g} / \mathrm{ml}$ in DMEM/F12) for 60 minutes at $37^{\circ} \mathrm{C}$ under agitation $(110 \mathrm{rpm})$ to dissociate interstitial tissue from seminiferous tubules. After centrifugation, the seminiferous tubule pellet was digested by trypsin $\left(0.25 \%, 5 \mathrm{ml} / \mathrm{g}, 20\right.$ minutes at $\left.37^{\circ} \mathrm{C}\right)$. Trypsin was inactivated, and cells were filtered $(60 \mu \mathrm{m})$ and cultured overnight in DMEM/F12 medium supplemented with $1 \times$ nonessential amino acids, $1 \times$ ITS, $100 \mathrm{U} / \mathrm{ml}$ penicillin, $100 \mu \mathrm{g} / \mathrm{ml}$ streptomycin, and 10\% FCS (all from Gibco). Primary testicular germ cells (TGCs) and Tcam-2 cells were incubated with ZIKV diluted in serum-free medium at an MOI of 1 (corresponding to $1.43 \times 10^{6} \mathrm{TCID}_{50} /$ million cells) for 2 hours at $37^{\circ} \mathrm{C}, 5 \% \mathrm{CO}_{2}$. Virus was removed by washing and trypsin treatment for 5 minutes at $37^{\circ} \mathrm{C}$. Primary testicular cells were cultured at a density of 0.5 million cells/ml in supplemented StemPro-34 (Invitrogen) as described elsewhere (67). T-cam 2 cells were cultured at a density of 0.1 million cells/ml in RPMI1640 supplemented with P/S, glutamine $(2 \mathrm{mM})$, and $10 \% \mathrm{FCS}$ (all reagents from Gibco).

Semen samples. Semen was liquified at $37^{\circ} \mathrm{C}$ for 30 minutes and 10 $\mu \mathrm{l}$ spread on a glass slide and dried at room temperature. Smears were fixed in $4 \%$ formaldehyde and stored at $-20^{\circ} \mathrm{C}$. Viral loads for $\mathrm{ZIKV}$ in seminal plasma and seminal cells were $7.25 \mathrm{log}$ copies $/ \mathrm{ml}$ and $6.7 \mathrm{log}$ copies/ $\mu$ g total RNA, respectively, for the donor on day 7, and $7.8 \mathrm{log}$ copies $/ \mathrm{ml}$ and $7.8 \mathrm{log}$ copies $/ 2 \times 10^{6}$ cells, respectively, for the donor on day 11. Patients' serology for dengue was negative, and semen samples tested negative for dengue by RT-PCR.

Mice. Mice lacking the type I IFN receptor (68) were backcrossed more than 10 times onto the C57BL/6 background (referred to as $\mathrm{IFNAR}^{-/-}$mice). Seven-week-old male $\mathrm{IFNAR}^{-/-}$mice were infected through the i.p. route with $10^{4} \mathrm{TCID}_{50} / 100 \mu \mathrm{l}$ of $\mathrm{ZIKV}(\mathrm{H} / \mathrm{PF} / 2013)$ or with PBS. Five and 9 days after infection, mice were sacrificed with carbon dioxide, and collected tissue was either frozen at $-80^{\circ} \mathrm{C}$ or fixed in PFA $4 \%$.

$R T-q P C R$. Total RNA was extracted using QIAamp vRNA (for supernatants) or RNeasy isolation kit (for tissue/cells) and treated with DNase (all from QIAGEN). Extracted RNA from explant supernatants was subjected to RT-qPCR using the GoTaq Probe 1-Step RT-qPCR System (Promega). Primers and probes for ZIKV described previously (69) were adapted as follows: ZIKV primer forward CCGCTGCCCAACACAAG, ZIKV primer reverse CCACTAACGTTCTTTTGCAGACAT, ZIKV probe AGCCTACCTTGACAAGCAATCAGACACTCAA. A standard curve with serial dilution of a known number of copies of vRNA was systematically run. The relative quantification of (a) steroidogenesis enzyme mRNA (STAR; CYP11A1, cytochrome P450 family 11 subfamily A member 1; HSD3B2, hydroxy-delta-5-steroid dehydrogenase 3 beta- and steroid delta-isomerase 2; HSD17B3, hydroxysteroid 17-beta dehydrogenase 3; CYP17A1, cytochrome P450 family 17 subfamily A member 1) and (b) testicular cell marker mRNA (inhibin B; Acta2, actin alpha 2 smooth muscle aorta; PGK2, phosphoglycerate kinase 2; PRM2, protamine) was performed as previously described (29).

Primers for innate immune response effector genes (Supplemental Table 1) were designed using the Primer-BLAST tool (70). Total RNA was reverse transcribed using the iScript cDNA Synthesis Kit (Bio-Rad). RT-qPCR reactions were performed on a BioRad CFX384 instrument using iTaq SYBR green mix (Bio-Rad) and 40 cycles of 15 seconds at $95^{\circ} \mathrm{C}$ and 1 minute at $60^{\circ} \mathrm{C}$, followed by melt-curve analysis. Gene expression fold changes were calculated with the $2^{-\Delta \Delta \mathrm{Ct}}$ method normalized to $\beta$-actin and mock-infected sample expression levels.

Determination of viral titer. VeroE6 cells seeded in opaque-walled 96-well plates at a final concentration of $1.5 \times 10^{5}$ cells $/ \mathrm{ml}$ in DMEM with $5 \%$ FCS were put in contact the next day with serial dilutions of supernatant. TCID $_{50} / \mathrm{ml}$ was measured on day 5 after infection using the Viral ToxGlo Assay (Promega).

Histology, RNAscope ISH, and IHC. Tissues or cell pellets were fixed in $4 \%$ formaldehyde and embedded in paraffin. RNA ISH was performed using RNAscope 2.5 (Advanced Cell Diagnostics) according to the manufacturer's instructions, as previously described (71, 72). RNAScope ISH is a highly specific and sensitive technique, with the ability to detect single molecules (72). Formaldehyde-fixed, paraffin-embedded tissue sections or cell pellets were deparaffinized in xylene and dehydrated in ethanol for 10 minutes at room temperature. Slides deparaffinized and $\mathrm{H}_{2} \mathrm{O}_{2}$ quenched for endogenous peroxidases were boiled in RNAscope Target Retrieval Reagents (citrate buffer $10 \mathrm{mM}, \mathrm{pH}$ 6, 15 minutes) and incubated in RNAscope Protease Plus $\left(40^{\circ} \mathrm{C}, 20 \mathrm{~min}-\right.$ utes), prior to probe hybridization. Sections were incubated with target probes ( 2 hours, $40^{\circ} \mathrm{C}$ ), washed in buffer, and incubated with amplification reagents. Chromogenic detection was performed using Fast Red as substrate for alkaline phosphatase to generate red signal. Slides were counterstained with hematoxylin and mounted in Eukitt (O. Kindler) before observation using bright-field microscopy. The "double Z" probes targeting ZIKV RNA (consensus sequence, target region 2195,443 , catalog 467771) and positive (targeting the 2514-3,433 region of human POLR2A gene, catalog 310451) and negative (targeting the 414862 region of bacterial $d a p B$ gene, catalog 310043) control probes were all obtained from Advanced Cell Diagnostics. Staining specificity was verified as shown in Supplemental Figure 3. Sections of ZIKV-infected Vero cell pellets and mock-infected testis tissues were systematically used as positive and negative controls.

Dual fluorescence ISH-IHC experiments was performed essentially as we previously described (71). Briefly, tissue sections were first submitted to ISH, then blocked in PBS/BSA $2 \%$ and incubated overnight at $4^{\circ} \mathrm{C}$ with primary antibody. Sections were washed, incubated with either anti-mouse or anti-rabbit Alexa Fluor 488 fluorescent secondary antibodies diluted 1:500 (chicken anti-mouse Alex Fluor 488 , product A-21200, and chicken anti-rabbit Alexa Fluor 488 product A-21441, Life Technologies), and counterstained with ProLong medium (Thermo Fisher Scientific) containing DAPI before observation with a Zeiss Axio Imager M1 fluorescence microscope connected to a digital camera using Zen software. Fluorescent Fast Red signal was read at $550 \mathrm{~nm}$.

Single IHC was performed as described previously (73). For immunofluorescence experiments, Alexa Fluor 488- or 594-coupled 
secondary antibodies diluted 1:500 (goat anti-mouse Alexa Fluor 594, product A-11032; chicken anti-rabbit Alexa Fluor 594, product A21442; donkey anti-rat Alexa Fluor 488, product A21208; all from Life Technologies) were used and sections mounted with ProLong DAPI to stain the nuclei. Cell staining was never observed for isotypic controls or mock-infected samples. Primary antibodies used were mouse anti-NS1 (BioFront Technologies, clone 0102136, $4 \mu \mathrm{g} / \mathrm{ml}$ ), anti-CD68 (Dako, clone KP1, $1.85 \mu \mathrm{g} / \mathrm{ml}$ ), anti-CD163 (Leica Novocastra, clone 10D6, 1:100), anti- $\alpha$-SMA (Dako, clone 1A4, 1,4 $\mu \mathrm{g}$ / $\mathrm{ml}$ ), anti-DDX4 (GeneTex, clone 2F9H5, 1:200), anti-ZO-1 (Thermo Fisher Scientific, clone ZO1-1A12, $10 \mu \mathrm{g} / \mathrm{ml}$ ); rabbit anti-Cyp11A1 (Sigma-Aldrich, 1:250), anti-cleaved caspase-3 (Cell Signaling Technology, Asp175, 1:50), anti-DDX4 (Abcam, $2 \mu \mathrm{g} / \mathrm{ml}$ ), anti-STAR (Cell Signaling Technology, 1:200), anti-CD3 (Dako, A0452, $10 \mu \mathrm{g} / \mathrm{ml}$ ), rat anti-mouse F4/80 (Abcam, clone BM8, 1:20).

The number of ZIKV $\mathrm{RNA}^{+} \mathrm{CD} 68 / \mathrm{CD} 163^{+}$macrophages, $\mathrm{CYP}_{11 \mathrm{~A}^{+}}$Leydig cells, $\alpha-\mathrm{SMA}^{+}$peritubular cells, germ cells, and Sertoli cells (identified on morphological criteria in light microscopy) in testis explants was assessed in 4 donors and at least 3 whole testis tissue sections/donor (corresponding to about $12 \mathrm{~mm}^{2} /$ testis donor). Quantification of $\mathrm{CD}^{+}$cells in mouse testis was performed in 3 mockinfected animals, 4 animals on day 5 p.i., and 4 animals on day 9 p.i., in at least $5 \mathrm{~mm}^{2} /$ testis. Slides were scanned with a NanoZoomer slide scanner (Hamamatsu Photonics, at Plateforme H2P2, Biosit). Immunostained positive cells were counted with NIH Image J software.

Immunocytofluorescence. Semen smears from donors and cell pellets from testicular cell cultures put onto polylysine-coated glass coverslips were fixed in $4 \%$ paraformaldehyde for 20 minutes at room temperature. After permeabilization (0.2\% Triton X-100, 10 minutes), the slides were incubated in blocking buffer $(0.2 \%$ Triton $\mathrm{X}-100,1 \%$ goat serum, 2 hours) and stained with antibodies against ZIKV NS1 (1:1,000, BioFront Technologies) or flavivirus envelope Ab 4G2 (1:1,000, Millipore). NS1 antibody was either directly coupled to Alexa Fluor 647 (Zenon labeling kit, Molecular Probes) or revealed using Alexa Fluor 555 goat anti-mouse (Life Technologies). Infected cell characterization was performed using rabbit anti-DDX4 $(5 \mu \mathrm{g} /$ $\mathrm{ml}$, Abcam, ab13840), anti-STRA8 $(9.6 \mu \mathrm{g} / \mathrm{ml}$, Thermo Fisher Scientific PA5-35047), and anti-FSHR (10 $\mu \mathrm{g} / \mathrm{ml}$, Origene, TA313897), detected using Alexa Fluor 488 (Invitrogen) or Alexa Fluor 647 goat anti-rabbit (Jackson ImmunoResearch Laboratories Inc.); mouse antiMAGEA-4 (clone 57B, $4 \mu \mathrm{g} / \mathrm{ml}$, provided by Giulio Spagnoli, Ludwig Institute for Cancer Research, Basel, Switzerland) coupled to Alexa Fluor 647 (Zenon labeling kit, Molecular Probes) and mouse anti- $\alpha$ SMA (clone 1A4, $0.5 \mu \mathrm{g} / \mathrm{ml}$, Dako, M0851) detected using Alexa Fluor 555 goat-anti mouse antibody. Isotype control antibodies or noninfected cells were used as negative controls. Slides were counterstained with ProLong medium containing DAPI. Images were acquired with the SP8 confocal system (Leica) connected to LAS software or with a DMRXA wide-field microscope (Applied Precision) and analyzed using Fiji software.

Testosterone and inhibin B immunoassays. Testosterone was assayed using a specific radioimmunoassay (Immunotech, Beckman Coulter). Inhibin B was assayed using a commercial ELISA kit (DSL10-84100 Active, Beckman Coulter).

Viability assay. Cell viability was assessed by measuring the release of LDH using the enzymatic fluorimetric assay CytoTox-ONE Homogeneous Membrane Integrity Assay (Promega).
Cytokine release measurement. A bead-based multiplex flow cytometry LEGENDplex assay (BioLegend, Ozyme) was used. Fluorescence was read using the BD LSRII Fortessa flow cytometer.

Statistics. Data were analyzed with nonparametric paired FriedmanDunn or unpaired Kruskal-Wallis-Dunn test when more than 2 sets of samples were compared, as specified in the figure legends. Nonparametric Mann-Whitney $U$ test was used to analyze differences in viral load of mice testis on days 5 and 9 after infection. Correlations were calculated using Spearman's test. Values were considered significant when $P$ was less than 0.05. Statistical analyses were performed using commercially available software (GraphPad Prism 6, GraphPad Software).

Study approval. Normal testes were obtained either after orchidectomy from prostate cancer patients who had not received any hormone therapy or at autopsy, and processed within 2 hours of surgery. The procedure was approved by Ethics Committee Ouest V, Rennes, France (authorization DC-2016-2783) and the French National Agency for Biomedical Research (authorization PF S09-015). Semen samples produced by masturbation were obtained from $2 \mathrm{ZIKV}$-infected donors living in the French Caribbean at 7 and 11 days after onset of symptoms, respectively, after written informed consent was obtained, in the French Cohort of Patients Infected by an Arbovirus (CARBO; ClinicalTrials.gov identifier NCT01099852). Mice were housed at the Institut Pasteur Animal Facility, accredited by the French Ministry of Agriculture for performing experiments on live rodents. Work on animals was performed in compliance with French and European regulations on the care and protection of laboratory animals (EC Directive 2010/63, French Law 2013-118, February 6,2013). All experiments with $\mathrm{IFNAR}^{-/-}$mice were approved by the Ethics Committee \#89 and registered under reference 2016-0018.

\section{Author contributions}

GM performed experiments, analyzed data, cowrote the manuscript, and contributed to data interpretation and study design. LH, APS, DM, FA, and ALT performed experiments, analyzed data, and contributed to writing the manuscript. LH designed primers and interpreted PCR array results. JF and SB performed experiments. GA interpreted data. $\mathrm{KB}$ and $\mathrm{SL}$ contributed testis tissues. GJ, LB, and AC contributed semen samples. TC and ML performed IFNAR mouse infection and tissue collection. NDR designed experiments, interpreted the data, and wrote the manuscript. All authors read, edited, and approved the manuscript.

\section{Acknowledgments}

This project received funding from the European Union's Horizon 2020 research and innovation program under grant agreement no. 733176, ZikaPLAN (grant agreement no. 734584), and ZIKAlliance (grant agreement 734548) and was funded in part by LabEx IBEID, Institut Pasteur, and Inserm. GM received support from REACTing. GA and NDR received funding from CAPES-COFECUB. GJ and LB received funding from Agence de la biomédecine and Agence régionale de santé Guadeloupe for the collection of semen from infected men. AC received funding from the French Ministry of Health (Soutien exceptionnel à la recherche et à l'innovation) and support from REACTing. Experiments were conducted in part on L3, MRic, and $\mathrm{H} 2 \mathrm{P} 2$ platforms at Biosit federative structure (Université de Rennes, CNRS, Inserm, Biosit [Biologie, Santé, Innovation Technologique de Rennes] - UMS 3480, US_S 018). This publication was supported by the European Virus Archive Goes Global (EVAg) project, which has 
received funding from the European Union's Horizon 2020 research and innovation program under grant agreement 653316. We thank Xavier Montagutelli for help with animal experiments and Laurianne Lesné and Christèle Desdoits for assistance in hormonal assays.
Address correspondence to: Nathalie Dejucq-Rainsford, IRSETInserm U1085, 9 avenue du Pr Léon Bernard, F-35000 Rennes, France. Phone: 33.2.2323.5069; Email: nathalie.dejucq-rainsford@ univ-rennes1.fr.
1. Coelho FC, et al. Higher incidence of Zika in adult women than adult men in Rio de Janeiro suggests a significant contribution of sexual transmission from men to women. Int J Infect Dis. 2016;51:128-132.

2. Yakob L, Kucharski A, Hue S, Edmunds WJ. Low risk of a sexually-transmitted Zika virus outbreak. Lancet Infect Dis. 2016;16(10):1100-1102.

3. Moreira J, Peixoto TM, Siqueira AM, Lamas CC. Sexually acquired Zika virus: a systematic review. Clin Microbiol Infect. 2017;23(5):296-305.

4. Duggal NK, et al. Frequent Zika virus sexual transmission and prolonged viral RNA shedding in an immunodeficient mouse model. Cell Rep. 2017;18(7):1751-1760.

5. Haddow AD, et al. High Infection rates for adult macaques after intravaginal or intrarectal inoculation with Zika virus. Emerging Infect Dis. 2017;23(8):1274-1281.

6. Carroll $\mathrm{T}$, et al. Zika virus preferentially replicates in the female reproductive tract after vaginal inoculation of rhesus macaques. PLoS Pathog. 2017;13(7):e1006537.

7. Tang WW, Young MP, Mamidi A, Regla-Nava JA, Kim K, Shresta S. A mouse model of Zika virus sexual transmission and vaginal viral replication. Cell Rep. 2016;17(12):3091-3098.

8. Duggal NK, McDonald EM, Ritter JM, Brault AC. Sexual transmission of Zika virus enhances in utero transmission in a mouse model. Sci Rep. 2018;8(1):4510.

9. Epelboin S, Dulioust E, Epelboin L, Benachi A, Merlet F, Patrat $\mathrm{C}$. Zika virus and reproduction: facts, questions and current management. Hum Reprod Update. 2017;23(6):629-645.

10. Barzon L, et al. Virus and antibody dynamics in travelers with acute Zika virus infection. Clin Infect Dis. 2018;66(8):1173-1180.

11. Mead PS, et al. Zika virus shedding in semen of symptomatic infected men. N Engl J Med. 2018;378(15):1377-1385.

12. Govero J, et al. Zika virus infection damages the testes in mice. Nature. 2016;540(7633):438-442.

13. Ma W, et al. Zika virus causes testis damage and leads to male infertility in mice. Cell. 2016;167(6):1511-1524.e10.

14. Griffin $\mathrm{BD}$, et al. DNA vaccination protects mice against Zika virus-induced damage to the testes. Nat Commun. 2017;8:15743.

15. Shan C, et al. A single-dose live-attenuated vaccine prevents Zika virus pregnancy transmission and testis damage. Nat Commun. 2017;8(1):676.

16. Osuna $\mathrm{CE}$, et al. Zika viral dynamics and shedding in rhesus and cynomolgus macaques. Nat Med. 2016;22(12):1448-1455.

17. Hirsch AJ, et al. Zika virus infection of rhesus macaques leads to viral persistence in multiple tissues. PLoS Pathog. 2017;13(3):e1006219.

18. Koide F, et al. Development of a Zika virus infection model in cynomolgus macaques. Front Microbiol. 2016;7:2028.

19. Joguet $G$, et al. Effect of acute Zika virus infection on sperm and virus clearance in body fluids: a prospective observational study. Lancet Infect Dis. 2017;17(11):1200-1208.

20. Roulet V, et al. Human testis in organotypic culture: application for basic or clinical research. Hum Reprod. 2006;21(6):1564-1575.

21. Young JC, et al. TCam-2 seminoma cell line exhibits characteristic foetal germ cell responses to TGF- $\beta$ ligands and retinoic acid. Int J Androl. 2011;34(4 pt 2):e204-e217.

22. Uraki R, et al. Zika virus causes testicular atrophy. SciAdv. 2017;3(2):e1602899.

23. Xia Y, Zhu WJ, Hao SF, Liang WB, Li J. Stereological analysis of age-related changes of testicular peritubular cells in men. Arch Gerontol Geriatr. 2012;55(1):116-119.

24. Nieschlag E, Behre HM, eds. Andrology. Berlin, Germany: Springer; 2010.

25. Robinson CL, et al. Male germ cells support longterm propagation of Zika virus. Nat Commun. 2018;9(1):2090.

26. Mansuy JM, et al. Zika virus in semen and spermatozoa. Lancet Infect Dis. 2016;16(10):1106-1107.

27. Siemann DN, Strange DP, Maharaj PN, Shi PY, Verma S. Zika virus infects human sertoli cells and modulates the integrity of the in vitro blood-testis barrier model. JVirol. 2017;91(22): e00623-17.

28. Kumar A, et al. Human Sertoli cells support high levels of Zika virus replication and persistence. Sci Rep. 2018;8(1):5477.

29. Kristensen DM, et al. Ibuprofen alters human testicular physiology to produce a state of compensated hypogonadism. Proc Natl Acad Sci U S A. 2018;115(4):E715-E724.

30. Adamopoulos DA, Lawrence DM, Vassilopoulos P, Contoyiannis PA, Swyer GI. Pituitary-testicular interrelationships in mumps orchitis and other viral infections. Br Med J. 1978;1(6121):1177-1180.

31. Potter SJ, DeFalco T. Role of the testis interstitial compartment in spermatogonial stem cell function. Reproduction. 2017;153(4):R151-R162.

32. Cheng CY, Wong EW, Yan HH, Mruk DD. Regulation of spermatogenesis in the microenvironment of the seminiferous epithelium: new insights and advances. Mol Cell Endocrinol. 2010;315(1-2):49-56.

33. Mayerhofer A. Human testicular peritubular cells: more than meets the eye. Reproduction 2013;145(5):R107-R116.

34. Huits R, De Smet B, Ariën KK, Van Esbroeck M, Bottieau E, Cnops L. Zika virus in semen: a prospective cohort study of symptomatic travellers returning to Belgium. Bull World Health Organ. 2017;95(12):802-809.

35. Sergerie M, Mieusset R, Croute F, Daudin M, Bujan L. High risk of temporary alteration of semen parameters after recent acute febrile illness. Fertil Steril. 2007;88(4):970.e1-970.e7.

36. Evenson DP, Jost LK, Corzett M, Balhorn R. Characteristics of human sperm chromatin structure following an episode of influenza and high fever: a case study. J Androl. 2000;21(5):739-746.
37. Quicke KM, et al. Zika virus infects human placental macrophages. Cell Host Microbe. 2016;20(1):83-90.

38. Mladinich MC, Schwedes J, Mackow ER. Zika virus persistently infects and is basolaterally released from primary human brain microvascular endothelial cells. MBio. 2017;8(4):e00952-17.

39. Strange DP, Green R, Siemann DN, Gale M, Verma S. Immunoprofiles of human Sertoli cells infected with Zika virus reveals unique insights into host-pathogen crosstalk. Sci Rep. 2018;8(1):8702.

40. Strange DP, et al. Human testicular organoid system as a novel tool to study Zika virus pathogenesis. Emerg Microbes Infect. 2018;7(1):82.

41. Singh PK, et al. Zika virus infects cells lining the blood-retinal barrier and causes chorioretinal atrophy in mouse eyes. JCI Insight. 2017;2(4):e92340.

42. Van der Hoek KH, et al. Viperin is an important host restriction factor in control of Zika virus infection. Sci Rep. 2017;7(1):4475.

43. Savidis G, et al. The IFITMs inhibit Zika virus replication. Cell Rep. 2016;15(11):2323-2330.

44. Perelygin AA, Scherbik SV, Zhulin IB, Stockman BM, Li Y, Brinton MA. Positional cloning of the murine flavivirus resistance gene. Proc Natl Acad Sci U S A. 2002;99(14):9322-9327.

45. Chen J, et al. Outcomes of congenital Zika disease depend on timing of infection and maternal-fetal interferon action. Cell Rep. 2017;21(6):1588-1599.

46. Schoggins JW, et al. A diverse range of gene products are effectors of the type I interferon antiviral response. Nature. 2011;472(7344):481-485.

47. Ngono AE, Shresta S. Immune response to dengue and Zika. Annu Rev Immunol. 2018;36:279-308.

48. Xia H, et al. An evolutionary NS1 mutation enhances Zika virus evasion of host interferon induction. Nat Commun. 2018;9(1):414.

49. Satie AP, et al. Excess type I interferon signaling in the mouse seminiferous tubules leads to germ cell loss and sterility. J Biol Chem. 2011;286(26):23280-23295.

50. Hasan M, et al. Trex1 regulates lysosomal biogenesis and interferon-independent activation of antiviral genes. Nat Immunol. 2013;14(1):61-71.

51. Sheng ZY, et al. Sertoli cells are susceptible to ZIKV infection in mouse testis. Front Cell Infect Microbiol. 2017;7:272.

52. Chan JFW, et al. Zika virus infection in dexamethasone-immunosuppressed mice demonstrating disseminated infection with multi-organ involvement including orchitis effectively treated by recombinant type I interferons. EBioMedicine. 2016;14:112-122.

53. Winkler CW, et al. Adaptive immune responses to Zika virus are important for controlling virus infection and preventing infection in brain and testes. JImmunol. 2017;198(9):3526-3535.

54. Dejucq N, Lienard MO, Guillaume E, Dorval I, Jégou B. Expression of interferons- $\alpha$ and $-\gamma$ in testicular interstitial tissue and spermatogonia of 
the rat. Endocrinology. 1998;139(7):3081-3087.

55. Le Tortorec A, et al. Antiviral responses of human Leydig cells to mumps virus infection or poly I:C stimulation. Hum Reprod. 2008;23(9):2095-2103.

56. Dejucq N, Liénard MO, Jégou B. Interferons and interferon-induced antiviral proteins in the testis. J Reprod Immunol. 1998;41(1-2):291-300.

57. Dejucq N, Chousterman S, Jégou B. The testicular antiviral defense system: localization, expression, and regulation of $2^{\prime} 5^{\prime}$ oligoadenylate synthetase, double-stranded RNA-activated protein kinase, and Mx proteins in the rat seminiferous tubule. J Cell Biol. 1997;139(4):865-873.

58. Lalle E, et al. Prolonged detection of dengue virus RNA in the semen of a man returning from Thailand to Italy, January 2018. Euro Surveill. 2018;23(18):18-00197.

59. Barbosa CM, et al. Yellow fever virus RNA in urine and semen of convalescent patient, Brazil. Emerging Infect Dis. 2018;24(1):176-178.

60. Bandeira AC, et al. Prolonged shedding of Chikungunya virus in semen and urine: a new perspective for diagnosis and implications for transmission. IDCases. 2016;6:100-103.

61. Armah HB, et al. Systemic distribution of West
Nile virus infection: postmortem immunohistochemical study of six cases. Brain Pathol. 2007;17(4):354-362.

62. Guérin B, Pozzi N. Viruses in boar semen: detection and clinical as well as epidemiological consequences regarding disease transmission by artificial insemination. Theriogenology. 2005;63(2):556-572.

63. Stassen L, Armitage CW, van der Heide DJ, Beagley KW, Frentiu FD. Zika virus in the male reproductive tract. Viruses. 2018;10(4):E198.

64. Spencer JL, et al. Replication of Zika virus in human prostate cells: a potential source of sexually transmitted virus. J Infect Dis. 2018;217(4):538-547.

65 . Houzet L, et al. Seminal simian immunodeficiency virus in chronically infected cynomolgus macaques is dominated by virus originating from multiple genital organs. JVirol. 2018;92(14): e00133-18.

66. Mizuno Y, Gotoh A, Kamidono S, Kitazawa S. [Establishment and characterization of a new human testicular germ cell tumor cell line (TCam-2)]. Nihon Hinyokika Gakkai Zasshi. 1993;84(7):1211-1218.

67. Sadri-Ardekani H, et al. Propagation of human spermatogonial stem cells in vitro. JAMA. 2009;302(19):2127-2134.

68. Müller U, et al. Functional role of type I and type II interferons in antiviral defense. Science. 1994;264(5167):1918-1921.

69. Lanciotti RS, et al. Genetic and serologic properties of Zika virus associated with an epidemic, Yap State, Micronesia, 2007. Emerging Infect Dis. 2008;14(8):1232-1239.

70. Ye J, Coulouris G, Zaretskaya I, Cutcutache I, Rozen S, Madden TL. Primer-BLAST: a tool to design target-specific primers for polymerase chain reaction. BMC Bioinformatics. 2012;13:134.

71. Matusali G, et al. Detection of simian immunodeficiency virus in semen, urethra, and male reproductive organs during efficient highly active antiretroviral therapy. J Virol. 2015;89(11):5772-5787.

72. Deleage C, Chan CN, Busman-Sahay K, Estes JD. Next-generation in situ hybridization approaches to define and quantify HIV and SIV reservoirs in tissue microenvironments. Retrovirology. 2018;15(1):4.

73. Roulet V, et al. Susceptibility of human testis to human immunodeficiency virus-1 infection in situ and in vitro. Am J Pathol. 2006;169(6):2094-2103. 